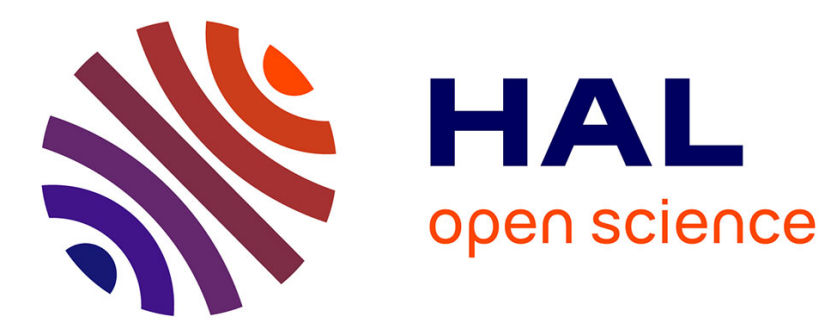

\title{
On the correlation structure of some random point processes on the line
}

Joel de Coninck, Francois Dunlop, Thierry Edmond Arnold Huillet

\section{To cite this version:}

Joel de Coninck, Francois Dunlop, Thierry Edmond Arnold Huillet. On the correlation structure of some random point processes on the line. Physica A: Statistical Mechanics and its Applications, 2008, 387 (5), pp.725-744. hal-00143863

\section{HAL Id: hal-00143863 https://hal.science/hal-00143863}

Submitted on 27 Apr 2007

HAL is a multi-disciplinary open access archive for the deposit and dissemination of scientific research documents, whether they are published or not. The documents may come from teaching and research institutions in France or abroad, or from public or private research centers.
L'archive ouverte pluridisciplinaire HAL, est destinée au dépôt et à la diffusion de documents scientifiques de niveau recherche, publiés ou non, émanant des établissements d'enseignement et de recherche français ou étrangers, des laboratoires publics ou privés. 


\title{
On the correlation structure of some random point processes on the line
}

\author{
Joël de Coninck ${ }^{(1)}$, François Dunlop ${ }^{(2)}$, Thierry Huillet ${ }^{(2)}$ \\ (1) Centre de Recherches en Modélisation Moléculaire, \\ Université de Mons-Hainaut, 20 Place du Parc, 7000 Mons, Belgium \\ (2) Laboratoire de Physique Théorique et Modélisation, \\ CNRS-UMR 8089 et Université de Cergy-Pontoise, \\ 2 Avenue Adolphe Chauvin, 95302, Cergy-Pontoise, France
}

\begin{abstract}
The correlation structure of some remarkable point processes on the onedimensional real line is investigated. More specifically, focus is on translation invariant determinantal, permanental and/or renewal point processes. In some cases, anomalous (non-Poissonian) fluctuations for the number of points in a large window can be observed. This may be read from the total correlation function of the point process. We try to understand when and why this occurs and what are the anomalous behaviors to be expected.

From examples, it is shown that determinantal (fermion) point processes can be super-homogeneous (the number variance grows slower than the number mean) and even hyper-uniform (when variance growth saturates).

Renewal point processes with bounded spacings variance are essentially Poissonian (the number variance grows like the number mean as in Poisson models).

Under certain conditions, permanental (boson) point processes can be subhomogeneous or critical (in the sense that the number variance grows faster than the number mean).

We give several detailed examples illustrating these properties of interest together with unexpected behaviors.
\end{abstract}

KEYWORDS: Random systems, determinantal, permanental and renewal point processes on the line, correlation functions, sub- and super-homogeneous point processes, anomalous fluctuations.

\section{Introduction}

In this note, we investigate the correlation structure of some peculiar point processes on the one-dimensional real line. We shall more precisely focus attention on translation invariant determinantal, permanental and/or renewal random point processes.

For 'essentially Poissonian' point processes, the number of points variance within a window grows like the number mean, as the size of the window grows large. This is typical of Poisson interaction-free gas which are ubiquitous in Nature.

In some cases of interest, anomalous (non-Poissonian) fluctuations for the random number of points in a large window arise. We try to understand when and why this 
occurs and what are the various anomalous behaviors to be expected. As will become apparent from the body of the text, these issues have recently been the source of considerable interest in the Physics literature.

It turns out that point processes can be 'super-homogeneous' (in that the number variance grows slower than the number mean) and even 'hyper-uniform' (when variance growth saturates). This expresses some degree of regularity of the model under study; ultimately, points could display a regularity close to the one of the standard lattice.

On the opposite, point processes can be 'sub-homogeneous' or critical (in the sense that the number variance grows faster than the number mean). Such point processes will exhibit large disorder.

The total correlation function of these random point processes may then be read from the number of points variance within a window. Anomalous fluctuations lead to anomalous correlations in that the structure factor exhibit unconventional behavior in a neighborhood of wave-length zero. We shall discuss this point and quantify these allegations.

From examples, it is shown that long-range determinantal (or fermion) point processes can be super-homogeneous and even hyper-uniform. Renewal point processes with bounded spacings variance and nearest-neighbor interactions are essentially Poissonian. Under certain conditions, permanental (boson) point processes can be sub-homogeneous or critical. We shall give an example.

After a brief introduction on random point processes with infinitely many particles on the line, we supply several detailed examples illustrating these properties of interest and we exhibit some of their unexpected behaviors.

\section{Generalities on random point processes (PP)}

To fix the background and notations, we start with generalities on random point processes.

\subsection{PP with a finite number of particles}

Given a system with $N$ indistinguishable particles, let $\mathbf{X}_{N}:=\left(X_{n} ; n=1, . ., N\right)$ denote their random position in $\mathbb{R}^{N}$. Assume that the probability density function (pdf) $u_{N}\left(\mathbf{x}_{N}\right)$ of their joint positions at point $\mathbf{x}_{N}:=\left(x_{1}, . ., x_{N}\right)$ of $\mathbb{R}^{N}$ exists. Under our assumption, $u_{N}\left(\mathbf{x}_{N}\right)$ is a symmetric (or exchangeable) function of its arguments. We shall also need to introduce $v_{N}\left(\mathbf{x}_{(N)}\right):=N ! \cdot u_{N}\left(\mathbf{x}_{(N)}\right)$ which is the joint pdf of $\mathbf{X}_{(N)}:=\left(X_{(n)} ; n=1, . ., N\right)$ at point $\mathbf{x}_{(N)}$, where $X_{(1)} \leq . . \leq X_{(N)}$ is the ordered version of $\mathbf{X}_{N}$. With $n<N$, let $u_{N}\left(\mathbf{x}_{n}\right):=\int_{\mathbb{R}^{N-n}} u_{N}\left(\mathbf{x}_{n} ; x_{n+1}, . ., x_{N}\right) d x_{n+1} . . d x_{N}$ stand for the $n$-point marginal density of $\mathbf{X}_{n}$. Let $\rho_{n, N}\left(\mathbf{x}_{n}\right):=\frac{N !}{(N-n) !} u_{N}\left(\mathbf{x}_{n}\right) \geq 0$ be the $n$-point correlation function (or points intensity) given that there are $N$ particles in the system. It is the probability density that there are particles at point $\mathbf{x}_{n}$. Clearly, $\rho_{n, N}\left(\mathbf{x}_{n}\right)$ is also symmetric. With $\mathbb{E}_{N}$ standing for the conditional expectation given $N$ particles, $\phi: \mathbb{R} \rightarrow \mathbb{C}$ some suitable test function, using exchangeability, we get

$$
\mathbb{E}_{N}\left(\prod_{n=1}^{N}\left(1+z \phi\left(X_{n}\right)\right)\right)=\sum_{n=0}^{N} z^{n}\left(\begin{array}{c}
N \\
n
\end{array}\right) \mathbb{E}\left(\prod_{m=1}^{n} \phi\left(X_{m}\right)\right)
$$




$$
\begin{gathered}
=\sum_{n=0}^{N} z^{n}\left(\begin{array}{c}
N \\
n
\end{array}\right) \int_{\mathbb{R}^{n}} \prod_{m=1}^{n} \phi\left(x_{m}\right) u_{N}\left(\mathbf{x}_{n}\right) d \mathbf{x}_{n} \\
=\sum_{n=0}^{N} \frac{z^{n}}{n !} \int_{\mathbb{R}^{n}} \prod_{m=1}^{n} \phi\left(x_{m}\right) \rho_{n, N}\left(\mathbf{x}_{n}\right) d \mathbf{x}_{n}
\end{gathered}
$$

where integration is with respect to the product Lebesgue measure $d \mathbf{x}_{n}:=d x_{1} . . d x_{n}$ in $\mathbb{R}^{n}$.

- If $z=1$ and $\phi(x)=\exp \{i \theta \varphi(x)\}-1$, where $\theta$ is a real parameter, the latter expansion reduces to

$$
\mathbb{E}_{N}\left(e^{i \theta \sum_{n=1}^{N} \varphi\left(X_{n}\right)}\right)=\sum_{n=0}^{N} \frac{1}{n !} \int_{\mathbb{R}^{n}} \prod_{m=1}^{n}\left(e^{i \theta \varphi\left(X_{m}\right)}-1\right) \rho_{n, N}\left(\mathbf{x}_{n}\right) d \mathbf{x}_{n}
$$

which is the usual generating function of the additive particle functional $\psi:=$ $\sum_{n=1}^{N} \varphi\left(X_{n}\right)$.

- Let $B \subseteq \mathbb{R}$ be a Borel set of $\mathbb{R}$. Let $\phi(x)=\mathbf{1}(x \in B)$, the indicator of $x$ in $B$. Then, with $\mathcal{N}_{N}(B):=\sum_{n=1}^{N} \mathbf{1}\left(X_{n} \in B\right)$, the number of points within $B$,

$$
\mathbb{E}_{N}\left(\prod_{n=1}^{N}\left(1+z \phi\left(X_{n}\right)\right)\right)=\mathbb{E}_{N}\left((1+z)^{\mathcal{N}_{N}(B)}\right)=\sum_{n=0}^{N} \frac{z^{n}}{n !} \int_{B^{n}} \rho_{n, N}\left(\mathbf{x}_{n}\right) d \mathbf{x}_{n}
$$

This shows that, with $\left[\mathcal{N}_{N}(B)\right]_{n}:=\frac{\mathcal{N}_{N}(B) !}{\left(\mathcal{N}_{N}(B)-n\right) !}$, the factorial moments of $\mathcal{N}_{N}(B)$ are given by:

$$
\mathbb{E}\left(\left[\mathcal{N}_{N}(B)\right]_{n}\right)=\int_{B^{n}} \rho_{n, N}\left(\mathbf{x}_{n}\right) d \mathbf{x}_{n} .
$$

Clearly, if $B=\mathbb{R}, \mathbb{E}\left(\left[\mathcal{N}_{N}(\mathbb{R})\right]_{n}\right)=\int_{\mathbb{R}^{n}} \rho_{n, N}\left(\mathbf{x}_{n}\right) d \mathbf{x}_{n}=\frac{N !}{(N-n) !}<\infty$ so that, as required, $\mathcal{N}_{N}(\mathbb{R}) \stackrel{d}{\sim} \delta_{N}$ (the Dirac law at point $\left.N\right)$.

Remark: if the components of $\mathbf{X}_{N}$ are independent and identically distributed (iid), then $u_{N}\left(\mathbf{x}_{N}\right)=\prod_{n=1}^{N} u_{1}\left(x_{n}\right)$ and $u_{N}\left(\mathbf{x}_{n}\right)=\prod_{m=1}^{n} u_{1}\left(x_{m}\right)$ does not depend on $N$. We get $\mathbb{E}_{N}\left((1+z)^{\mathcal{N}_{N}(B)}\right)=\left\{1+z \mathbb{P}\left(X_{1} \in B\right)\right\}^{N}$ so that $\mathcal{N}_{N}(B)$ has bino$\operatorname{mial} \operatorname{bin}\left(N, \mathbb{P}\left(X_{1} \in B\right)\right)$ distribution. For rare sets $B=B_{N}$ for which $\mathbb{P}\left(X_{1} \in B_{N}\right)=$ $\rho / N, \rho>0, \mathcal{N}_{N}\left(B_{N}\right) \stackrel{d}{\rightarrow} \operatorname{Poisson}(\rho)$ as $N \nearrow \infty . \diamond$

\section{Examples:}

- Let $\left(\psi_{i}(x) ; i=1, \ldots, N\right)$ be $N$ square-integrable real-valued functions satisfying $-\infty<\int_{\mathbb{R}} \psi_{i}(x) \psi_{j}(x) d x=a_{i, j}<\infty, i, j=1, . ., N$. Let $A_{N}=\left[a_{i, j}\right]$ be the square $N \times N$ matrix with entries $a_{i, j}$ and assume $A$ is not singular. With $\mathbf{x}_{N}=\left(x_{1}, \ldots, x_{N}\right)$, define the $N \times N$ weight matrix $W_{N}\left(\mathbf{x}_{N}\right)$ to be the symmetric matrix with $m \times m^{\prime}$ entry

$$
W_{N}\left(\mathbf{x}_{N}\right)_{m, m^{\prime}}=W_{N}\left(x_{m}, x_{m^{\prime}}\right)=\sum_{i, j=1}^{N} \psi_{i}\left(x_{m}\right)\left(A^{-1}\right)_{i, j} \psi_{j}\left(x_{m^{\prime}}\right), m, m^{\prime}=1, . ., N
$$


Let $\left|W_{N}\right|$ stand for the determinant of the matrix $W_{N}$. Let $\Psi_{N}\left(\mathbf{x}_{N}\right)$ be the $N \times$ $N$ matrix with $i \times j$-entry $\Psi_{N}\left(\mathbf{x}_{N}\right)_{i, j}=\psi_{i}\left(x_{j}\right)$. Then, observing $W_{N}\left(\mathbf{x}_{N}\right)=$ $\Psi_{N}\left(\mathbf{x}_{N}\right)^{\prime} A^{-1} \Psi_{N}\left(\mathbf{x}_{N}\right),\left|W_{N}\left(\mathbf{x}_{N}\right)\right|=\left|A^{-1}\right|\left|\Psi_{N}\left(\mathbf{x}_{N}\right)\right|^{2}$ and $\int_{\mathbb{R}^{N}}\left|\Psi_{N}\left(\mathbf{x}_{N}\right)\right|^{2} d \mathbf{x}_{N}=$ $N !|A|$, we get that

$$
u_{N}\left(\mathbf{x}_{N}\right)=\frac{1}{N !}\left|W_{N}\left(\mathbf{x}_{N}\right)\right|
$$

is a symmetric pdf on $\mathbb{R}^{N}$. Further,

$$
\rho_{n, N}\left(\mathbf{x}_{n}\right)=\left|W_{n, N}\left(\mathbf{x}_{n}\right)\right|
$$

where $W_{n, N}\left(\mathbf{x}_{n}\right)$ is the $n \times n$ matrix with entries $W_{n, N}\left(\mathbf{x}_{N}\right)_{m, m^{\prime}}=W_{n, N}\left(x_{m}, x_{m^{\prime}}\right)$ and

$$
W_{n, N}\left(x_{m}, x_{m^{\prime}}\right)=\sum_{i, j=1}^{N} \psi_{i}\left(x_{m}\right)\left(A^{-1}\right)_{i, j} \psi_{j}\left(x_{m^{\prime}}\right), m, m^{\prime}=1, . ., n .
$$

Such point processes with determinantal correlation functions are called determinantal with $N$ particles.

If $\left(\psi_{i}(x) ; i=1, . ., N\right)$ forms an orthonormal system, $\int_{\mathbb{R}} \psi_{i}(x) \psi_{j}(x) d x=\delta_{i, j}$ and

$$
W_{N}\left(\mathbf{x}_{N}\right)_{m, m^{\prime}}=W_{N}\left(x_{m}, x_{m^{\prime}}\right)=\sum_{i=1}^{N} \psi_{i}\left(x_{m}\right) \psi_{i}\left(x_{m^{\prime}}\right), m, m^{\prime}=1, . ., N
$$

A familiar example arises from eigenvalues distribution in the GUE ensemble of Random Matrix Theory. In this case, $\psi_{i}(x)=P_{i-1, N}(x) e^{-x^{2} / 2}$ where $P_{i-1, N}(x)$; $i=1, . ., N$ are the $N$ first orthonormal Hermite polynomials. The joint distribution of the ordered eigenvalues is

$$
v_{N}\left(\mathbf{x}_{(N)}\right)=\left|W_{N}\left(\mathbf{x}_{(N)}\right)\right|=\prod_{1 \leq m_{1}<m_{2} \leq N}\left(x_{\left(m_{2}\right)}-x_{\left(m_{1}\right)}\right)^{2} e^{-\sum_{m=1}^{N} x_{(m)}^{2}} .
$$

It is of interest to ask whether the kernel $W_{N}$ has a proper limit when $N \nearrow \infty$. It does but it requires scaling. For instance, using asymptotic formulae for Hermite polynomials, it may be shown that, with $z_{N}$ satisfying $W_{N}\left(z_{N}, z_{N}\right) \sim \sqrt{2 N} / \pi$ :

$\frac{\pi}{\sqrt{2 N}} W_{N}\left(z_{N}+\frac{\pi x_{(m)}}{\sqrt{2 N}}, z_{N}+\frac{\pi x_{\left(m^{\prime}\right)}}{\sqrt{2 N}}\right) \rightarrow_{N} \nearrow_{\infty} W\left(x_{m}, x_{m^{\prime}}\right)=\frac{\sin \left(\pi\left(x_{\left(m^{\prime}\right)}-x_{(m)}\right)\right)}{\pi\left(x_{\left(m^{\prime}\right)}-x_{(m)}\right)}$,

with a limiting sine kernel in the bulk of the spectrum as the number of 'particles' tends to infinity.

Similarly, by scaling around the largest eigenvalue,

$$
\begin{aligned}
& \frac{1}{\sqrt{2} N^{1 / 6}} W_{N}\left(\frac{2 N+N^{1 / 3} x_{(m)}}{\sqrt{2 N}}, \frac{2 N+N^{1 / 3} x_{\left(m^{\prime}\right)}}{\sqrt{2 N}}\right) \\
\rightarrow & N \nearrow \infty W\left(x_{(m)}, x_{\left(m^{\prime}\right)}\right)=A i\left(x_{m}, x_{m^{\prime}}\right)
\end{aligned}
$$

with a limiting Airy kernel $A i(.,$.$) at the edge of the spectrum (see [TW]). In sharp$ contrast to the previous limiting point process with a sine kernel, the limiting Airy point process is not translation invariant. This suggests that random point processes with infinitely many particles should be of interest. Before dealing with this point, 
let us first randomize the number of points in a PP with finitely many points.

Randomizing the number of points: Assume the number of points in the system is now random, let us call it $\mathcal{N}$. Assume $\mathcal{N}$ is finite almost surely (a.s.) and that $\mathcal{N}$ has all its moments finite. Then

$$
\begin{gathered}
\mathbb{E}\left(\prod_{N=1}^{\mathcal{N}}\left(1+z \phi\left(X_{N}\right)\right)\right)=\sum_{N \geq 0} \mathbb{P}(\mathcal{N}=N) \mathbb{E}_{N}\left(\prod_{n=1}^{N}\left(1+z \phi\left(X_{n}\right)\right)\right) \\
=\sum_{N \geq 0} \mathbb{P}(\mathcal{N}=N) \sum_{n=0}^{N} \frac{z^{n}}{n !} \int_{\mathbb{R}^{n}} \prod_{m=1}^{n} \phi\left(x_{m}\right) \rho_{n, N}\left(\mathbf{x}_{n}\right) d \mathbf{x}_{n} \\
=\sum_{n \geq 0} \frac{z^{n}}{n !} \sum_{N \geq n} \int_{\mathbb{R}^{n}} \prod_{m=1}^{n} \phi\left(x_{m}\right) \rho_{n}\left(\mathbf{x}_{n} ; N\right) d \mathbf{x}_{n}
\end{gathered}
$$

where

$$
\rho_{n}\left(\mathbf{x}_{n} ; N\right)=\mathbb{P}(\mathcal{N}=N) \rho_{n, N}\left(\mathbf{x}_{n}\right) .
$$

Thus,

$$
\mathbb{E}\left(\prod_{N=1}^{\mathcal{N}}\left(1+z \phi\left(X_{N}\right)\right)\right)=\sum_{n \geq 0} \frac{z^{n}}{n !} \int_{\mathbb{R}^{n}} \prod_{m=1}^{n} \phi\left(x_{m}\right) \rho_{n}\left(\mathbf{x}_{n}\right) d \mathbf{x}_{n}
$$

where now

$$
\rho_{n}\left(\mathbf{x}_{n}\right):=\sum_{N \geq n} \rho_{n}\left(\mathbf{x}_{n} ; N\right) .
$$

The function $\rho_{n}\left(\mathbf{x}_{n}\right)$ is called the $n$-point correlation function of $\left(X_{N} ; N=1, \ldots, \mathcal{N}\right)$. Clearly

$$
\begin{aligned}
\rho_{n}\left(\mathbf{x}_{n}\right) & =\sum_{N \geq n} \frac{N !}{(N-n) !} \mathbb{P}(\mathcal{N}=N) u_{N}\left(\mathbf{x}_{n}\right) \\
& =\sum_{N \geq n} \frac{N !}{(N-n) !} u\left(\mathbf{x}_{n} ; N\right)
\end{aligned}
$$

where

$$
u\left(\mathbf{x}_{n} ; N\right)=\mathbb{P}(\mathcal{N}=N) u_{N}\left(\mathbf{x}_{n}\right)=\int_{\mathbb{R}^{N-n}} u_{N}\left(\mathbf{x}_{n} ; x_{n+1}, . ., x_{N}\right) d x_{n+1} . . d x_{N}
$$

is the joint probability that there are $N$ particles in the system and that the $n$ first one are located at points $\mathbf{x}_{n}$.

Let $\phi(x)=\mathbf{1}(x \in \mathbb{R})$. Then

$$
\mathbb{E}\left((1+z)^{\mathcal{N}}\right)=\sum_{n \geq 0} \frac{z^{n}}{n !} \int_{\mathbb{R}^{n}} \rho_{n}\left(\mathbf{x}_{n}\right) d \mathbf{x}_{n}
$$

and, since these quantities exist

$$
\mathbb{E}\left([\mathcal{N}]_{n}\right)=\int_{\mathbb{R}^{n}} \rho_{n}\left(\mathbf{x}_{n}\right) d \mathbf{x}_{n}=\sum_{N \geq n} \frac{N !}{(N-n) !} \mathbb{P}(\mathcal{N}=N)<\infty .
$$


Further,

$$
\begin{aligned}
& \mathbb{E}\left(v^{\mathcal{N}}\right)=\sum_{n \geq 0} \frac{(v-1)^{n}}{n !} \int_{\mathbb{R}^{n}} \rho_{n}\left(\mathbf{x}_{n}\right) d \mathbf{x}_{n} \\
& =\sum_{m \geq 0} \frac{v^{m}}{m !} \sum_{n \geq m} \frac{(-1)^{n-m}}{(n-m) !} \int_{\mathbb{R}^{n}} \rho_{n}\left(\mathbf{x}_{n}\right) d \mathbf{x}_{n}
\end{aligned}
$$

showing that

$$
\mathbb{P}(\mathcal{N}=m)=\frac{1}{m !} \sum_{n \geq m} \frac{(-1)^{n-m}}{(n-m) !} \int_{\mathbb{R}^{n}} \rho_{n}\left(\mathbf{x}_{n}\right) d \mathbf{x}_{n} .
$$

\subsection{PP with an infinite number of particles}

Assume $\mathcal{N}:=\mathcal{N}(\mathbb{R})=\infty$ with probability 1 so that the total number of points is now infinite. Suppose however that, for each bounded subset $B$ of $\mathbb{R}$ with finite volume $|B|, \mathcal{N}(B)<\infty$, a.s.. Then, the correlation functions at least need to be symmetric, non-negative and locally integrable on each $B$.

Reproducing the previous steps locally, for each bounded subset $B$ of $\mathbb{R}$, each bounded $\phi$ on $B$, if there is a maximal $z_{c}>0$ for which the finiteness condition

$$
\sum_{n \geq 0} \frac{\left(z_{c}\|\phi\|_{\infty}\right)^{n}}{n !} \int_{B^{n}} \rho_{n}\left(\mathbf{x}_{n}\right) d \mathbf{x}_{n}<\infty
$$

is fulfilled, then, for all $z \leq z_{c}$ :

$$
\mathbb{E}\left(\prod_{N=1}^{\mathcal{N}(B)}\left(1+z \phi\left(X_{N}\right)\right)\right)=\sum_{n \geq 0} \frac{z^{n}}{n !} \int_{B^{n}} \prod_{m=1}^{n} \phi\left(x_{m}\right) \rho_{n}\left(\mathbf{x}_{n}\right) d \mathbf{x}_{n}
$$

where

$$
\begin{aligned}
\rho_{n}\left(\mathbf{x}_{n}\right) & =\sum_{N \geq n} \frac{N !}{(N-n) !} \mathbb{P}(\mathcal{N}(B)=N) u_{N}\left(\mathbf{x}_{n}\right) \\
& =\sum_{N \geq n} \frac{N !}{(N-n) !} \int_{B^{N-n}} u_{N}\left(\mathbf{x}_{n} ; x_{n+1}, . ., x_{N}\right) d x_{n+1} . . d x_{N} .
\end{aligned}
$$

The finiteness condition is fulfilled for instance if $\int_{B^{n}} \rho_{n}\left(\mathbf{x}_{n}\right) d \mathbf{x}_{n}<C_{B}^{n}$, for some constant $C_{B}>0$ only depending on $B$.

In slightly different words, with $\left(\mathbf{x}_{N} \in B^{N}\right)_{N>1}$, if $\mathbf{x}_{(N)}=\left(x_{(1)}, . ., x_{(N)}\right)$ where $x_{(1)} \leq . . \leq x_{(N)}$ is the ordered version of $\mathbf{x}_{N}$ within $B$,

$$
\rho_{n}\left(\mathbf{x}_{(n)}\right)=\sum_{N \geq n} \frac{1}{(N-n) !} \int_{B^{N-n}} v_{N}\left(\mathbf{x}_{(n)} ; x_{(n+1)}, . ., x_{(N)}\right) d x_{(n+1)} . . d x_{(N)}
$$

is now the probability density that there are particles at $\mathbf{x}_{(n)}$ in $B$.

By inclusion-exclusion principle, this system can be inverted to give the distribution of $\mathbf{X}_{(n)}$ within $B$ as

$$
v_{n}\left(\mathbf{x}_{(n)}\right)=\sum_{N \geq n} \frac{(-1)^{N-n}}{(N-n) !} \int_{B^{N-n}} \rho_{N}\left(\mathbf{x}_{(n)} ; x_{(n+1)}, . ., x_{(N)}\right) d x_{(n+1)} . . d x_{(N)} .
$$


Assuming $B=\left[x_{(1)}, x_{(n)}\right]$

$$
v_{n}\left(\mathbf{x}_{(n)}\right)=\sum_{N \geq n} \frac{(-1)^{N-n}}{(N-n) !} \int_{\left[x_{(1)}, x_{(n)}\right]^{N-n}} \rho_{N}\left(\mathbf{x}_{(n)} ; x_{(n+1)}, . ., x_{(N)}\right) d x_{(n+1)} . . d x_{(N)}
$$

is the (Janossy) probability density that the $m$-th left-most particle is at $x_{(m)}$, $m=1, . ., n$ with no other particle in $\left[x_{(1)}, x_{(n)}\right]$.

Remark: The PP with correlation function $\rho_{n}\left(\mathbf{x}_{(n)}\right)$ is said to be translation invariant if, for each $n \geq 1$, each $x \in \mathbb{R}: \rho_{n}\left(\mathbf{x}_{(n)}\right)=\rho_{n}\left(x_{(1)}+x, x_{(2)}+x, . ., x_{(n)}+x\right)$. For translation invariant systems, choosing $x=-x_{(1)}$ we have:

$$
\rho_{n}\left(\mathbf{x}_{(n)}\right)=\rho_{n}\left(0, x_{(2)}-x_{(1)}, . ., x_{(n)}-x_{(1)}\right) .
$$

In particular, $\rho_{1}\left(\mathbf{x}_{(1)}\right)=\rho$ (the density of points is constant),

$$
\rho_{2}\left(\mathbf{x}_{(2)}\right)=\rho_{2}\left(x_{(2)}-x_{(1)}\right)=: \rho^{2} g\left(x_{(2)}-x_{(1)}\right), \ldots
$$

For translation invariant PP, the effect of a Bernoulli thinning operation is simple: by thinning, we mean that each point of the translation invariant PP is erased with probability $p \in(0,1)$ and left unchanged with probability $1-p$, independently for each point, the erosion process being independent of randomness entering into the construction of the initial PP. Under these hypotheses, the correlations structure of the thinned process are the same as the one of the original process at the only exception that the original intensity of points $\rho$ is changed into $\rho p$. $\diamond$

Counting: Assuming $\phi(x)=\sum_{l=1}^{k} z_{l} \cdot \mathbf{1}\left(x \in B_{l}\right)$ where the $B_{l}$ are disjoint subsets of $B$, with $\mathcal{N}\left(B_{l}\right)=\sum_{N=1}^{\mathcal{N}(B)} \mathbf{1}\left(X_{N} \in B_{l}\right)$

$$
\begin{aligned}
& \mathbb{E}\left(\prod_{N=1}^{\mathcal{N}(B)}\left(1+z \phi\left(X_{N}\right)\right)\right)=\mathbb{E}\left(\prod_{l=1}^{k}\left(1+z \cdot z_{l}\right)^{\mathcal{N}\left(B_{l}\right)}\right) \\
& =\sum_{n \geq 0} \frac{z^{n}}{n !} \sum_{n_{1}+. .+n_{k}=n} \prod_{l=1}^{k} z_{l}^{n_{l}} \int_{B_{1}^{n_{1}} \times \ldots \times B_{k}^{n_{k}}} \rho_{n}\left(\mathbf{x}_{n}\right) d \mathbf{x}_{n},
\end{aligned}
$$

showing that, for each $n$ and $k$, each integers $n_{1}, . ., n_{k}$ such that $n_{1}+. .+n_{k}=n$

$$
\mathbb{E}\left(\prod_{l=1}^{k}\left[\mathcal{N}\left(B_{l}\right)\right]_{n_{l}}\right)=\int_{B_{1}^{n_{1}} \times . . \times B_{k}^{n_{k}}} \rho_{n}\left(\mathbf{x}_{n}\right) d \mathbf{x}_{n} .
$$

In particular, if $k=1, \mathbb{E}\left([\mathcal{N}(B)]_{n}\right)=\int_{B^{n}} \rho_{n}\left(\mathbf{x}_{n}\right) d \mathbf{x}_{n}$ and the variance $\sigma_{B}^{2}$ of $\mathcal{N}(B)$ is

$$
\sigma_{B}^{2}=\int_{B} \rho_{1}\left(x_{1}\right) d x_{1}-\int_{B^{2}}\left(\rho_{1}\left(x_{1}\right) \rho_{1}\left(x_{2}\right)-\rho_{2}\left(x_{1}, x_{2}\right)\right) d x_{1} d x_{2} .
$$


Right-most particle in $B$ : Assume $z=-1$ and $\phi(x)=\mathbf{1}\left(x>x_{*}\right)$ with $x_{*} \in \mathbb{R}$. Then

$$
\mathbb{E}\left(\prod_{N=1}^{\mathcal{N}(B)}\left(1+z \phi\left(X_{N}\right)\right)\right)=\mathbb{P}\left(X_{(\mathcal{N}(B))} \leq x_{*}\right)
$$

where $X_{(\mathcal{N}(B))}$ is the right-most particle in $B$. The distribution of $X_{(\mathcal{N}(B))}$ is given by

$$
\mathbb{P}\left(X_{(\mathcal{N}(B))} \leq x_{*}\right)=\sum_{n \geq 0} \frac{(-1)^{n}}{n !} \int_{\left[B \cap\left(x_{*}, \infty\right)\right]^{n}} \rho_{n}\left(\mathbf{x}_{n}\right) d \mathbf{x}_{n}
$$

If for all $x_{*} \in \mathbb{R}, \sum_{n \geq 0} \frac{(-1)^{n}}{n !} \int_{\left(x_{*}, \infty\right)^{n}} \rho_{n}\left(\mathbf{x}_{n}\right) d \mathbf{x}_{n}<\infty$, the PP is said to have a right-most particle. If this is the case, the latter quantity is its distribution function.

\section{Translation invariant examples}

We now give translation invariant examples of interest.

\subsection{Translation invariant determinantal (or fermion) point processes}

Construction, definitions and properties: With $f(r)=f(-r)$ satisfying $0<f(0)<\infty$, let $f(r) \in L^{2}(\mathbb{R})$ be a real positive-definite (or of positive type) function, meaning: for all integer $n$, all real numbers $\mathbf{x}_{n}:=\left(x_{m}, m=1, . ., n\right)$ and all $\mathbf{z}_{n} \in \mathbb{C}^{n}: \mathbf{z}_{n}^{\prime} \cdot W\left(\mathbf{x}_{n}\right) \cdot \mathbf{z}_{n} \geq 0$. Here $W\left(\mathbf{x}_{n}\right):=\left[f\left(x_{m}-x_{m^{\prime}}\right)\right]_{1}^{n}$ is the $n \times n$ symmetric matrix whose $m \times m^{\prime}$ entry is $W\left(\mathbf{x}_{n}\right)_{m, m^{\prime}}=W\left(x_{m}, x_{m^{\prime}}\right)=f\left(x_{m}-x_{m^{\prime}}\right)$, $m, m^{\prime} \in\{1, \ldots, n\}$.

We shall also let $W(\mathbf{x}):=\left[f\left(x_{m}-x_{m^{\prime}}\right)\right]_{1}^{\infty}$, an infinite dimensional weight matrix.

If $f$ is continuous, by Bochner theorem, positive-definiteness of $f$ holds if $\widehat{f}(\lambda):=$ $\int_{\mathbb{R}} e^{-i \lambda r} f(r) d r$ (which is real) is non-negative for almost all $\lambda$. Note therefore that $f(r)=\frac{1}{2 \pi} \int_{\mathbb{R}} e^{i \lambda r} \widehat{f}(\lambda) d \lambda$ is the Fourier transform of the bounded positive spectral density $\widehat{f}$ and $|f(r) / f(0)| \leq 1$. For each compact set $B \subset \mathbb{R}$, consider $W$ as the operator acting on $L^{2}(B)$ with kernel $W\left(x_{m}, x_{m^{\prime}}\right)$. Under our assumptions, by Mercer theorem, $W$ is locally trace class. Let $\left(\lambda_{k}(B) ; k \geq 0\right)$ be its corresponding eigenvalues. When $B$ is an interval, $\lambda_{k}(B)$ is also the $k$-th Fourier coefficient in the series expansion of $f(r)$ restricted to $B$. Then, the point process with determinantal correlation functions

$$
\rho_{n}\left(\mathbf{x}_{n}\right)=\left|W\left(\mathbf{x}_{n}\right)\right|, n \geq 1
$$

is well-defined and translation invariant, if and only if, for each $B$, each $k, \lambda_{k}(B) \in$ $[0,1]$. It is called a determinantal (or fermion) point process (for short DPP). See [DVJ], page $142,[\mathrm{M}]$ and $[\mathrm{S}]$. If this condition is to be fulfilled, necessarily, $\widehat{f}(\lambda) \in[0,1]$.

Let $P$ be the permutation matrix which maps the indices of $\mathbf{x}_{n}$ into the ones of $\mathbf{x}_{(n)}$ where now the $x_{(m)}$ are ordered on the real line. By considering instead the novel congruent matrix $P^{\prime} W_{n}\left(\mathbf{x}_{n}\right) P$, we also get the correlation functions

$$
\rho_{n}\left(\mathbf{x}_{(n)}\right)=\left|W\left(\mathbf{x}_{(n)}\right)\right|=\left|\left[f\left(x_{(m)}-x_{\left(m^{\prime}\right)}\right)\right]_{1}^{n}\right|, n \geq 1 .
$$


For instance, $\rho_{1}\left(\mathbf{x}_{(1)}\right)=f(0)=: \rho, \rho_{2}\left(\mathbf{x}_{(2)}\right)=\rho^{2} g\left(x_{(2)}-x_{(1)}\right)$ where, with $r>0$, $g(r):=1-\{f(r) / f(0)\}^{2} \in[0,1]$ are the first two correlation functions. We shall also use $h(r):=g(r)-1$ which is the total (centered) correlation function.

Remark: The set of positive-definite functions forms a closed cone as this property is preserved under convex linear combinations and point-wise products and if a sequence $\left(f_{k} ; k \geq 1\right)$ of such positive-definite functions converges, then the limit remains positive-definite.

If $f_{1}$ and $f_{2}$ are 2 positive-definite functions with associated weight matrices $W_{i}(\mathbf{x})=\left[f_{i}\left(x_{m}-x_{m^{\prime}}\right)\right], i=1,2$, then the weight matrix $W(\mathbf{x})$ associated to $f=f_{1} \cdot f_{2}$ is $W(\mathbf{x})=W_{1}(\mathbf{x}) \circ W_{2}(\mathbf{x})$ where $\circ$ stands for Hadamard (or Schur) entry-wise product. The Hadamard product of positive-definite matrices indeed is a positive-definite matrix. Taking $f_{1}(r)=f_{2}(r)=f(r) / f(0)$ where $f$ is definitepositive, it follows that $-h(r)$ is definite positive (or $h(r)$ is definite negative). For determinantal point processes, the total correlation function $h(r)$ is definite negative and negative (doubly negative), with values in $[-1,0] . \diamond$

There are two immediate consequences of this construction:

- When evaluating the limiting variance of the random number of points in an interval of length $x$ (as $x \nearrow \infty$ ) around the origin, the question of the integrability of $h(r)$ pops in. Integrability of $h(r)$ holds if and only if $f$ is square-integrable. When $f$ is integrable, this will be the case if $\widehat{f}(\lambda)$ itself is integrable and, if both functions are integrable, by Fourier-Plancherel theorem

$$
-f(0)^{2} \int_{\mathbb{R}} h(r) d r=\int_{\mathbb{R}} f(r)^{2} d r=\int_{\mathbb{R}} \widehat{f}(\lambda)^{2} d \lambda .
$$

- Finally, the generating functional of determinantal point processes takes a familiar form. For instance, in this case,

$$
\begin{aligned}
\mathbb{E}\left(\prod_{N=1}^{\mathcal{N}(B)}\left(1+z \phi\left(X_{N}\right)\right)\right) & =\sum_{n \geq 0} \frac{z^{n}}{n !} \int_{B^{n}} \prod_{m=1}^{n} \phi\left(x_{m}\right) \cdot\left|W\left(\mathbf{x}_{n}\right)\right| d \mathbf{x}_{n} \\
& =:|I+z W \phi|_{L^{2}(B)}
\end{aligned}
$$

and the expansion reduces to a Fredholm determinant where $W \phi$ is the operator on $L^{2}(B)$ with kernel $W\left(x_{m}, x_{m^{\prime}}\right) \phi\left(x_{m^{\prime}}\right)$. Similarly, for determinantal point processes, the right-most particle in $B$ distribution is

$$
\mathbb{P}\left(X_{(\mathcal{N}(B))} \leq x_{*}\right)=|I-W|_{L^{2}\left(B \cap\left(x_{*}, \infty\right)\right)} .
$$

Let us now give some examples.

\section{Examples:}


1./ With $\rho, \xi>0$, take for example $f(r)=\rho e^{-|r| /(2 \xi)}$, with $\widehat{f}(\lambda)=\frac{\rho \xi^{-1}}{(2 \xi)^{-2}+\lambda^{2}}$. The function $f$ is integrable and square-integrable. Further, $\widehat{f}(\lambda) \in[0,4 \rho \xi]$. Therefore, if $\rho$ belongs to some range $R \subseteq\left(0, \rho_{c}:=1 /(4 \xi)\right]$, the induced determinantal point process is well-defined and translation invariant with $g(r):=1-e^{-|r| / \xi}$. Here, $|h(r)|$ decays exponentially and $\int_{\mathbb{R}} h(r) d r=-2 \xi$ where $\xi$ is the correlation length. A broader class to which this Example belongs, corresponds to the choice $f(r)=$ $\rho e^{-\zeta\left|r / r_{0}\right|^{\alpha} / 2}$ where $\rho, \zeta, r_{0}>0$ and $\alpha \in(0,2]$. In that case, $h(r)=-e^{-\zeta\left|r / r_{0}\right|^{\alpha}}$. The Fourier transform of $f(r)$ is proportional to $\alpha$-stable pdf on the real line.

2./ With $\rho \in R \subseteq\left(0, \rho_{c}:=1\right]$, take for example $f(r)=\rho \sin (\pi r) /(\pi r)$, for which $\widehat{f}(\lambda)=\rho \cdot \mathbf{1}(\lambda \in[-\pi,+\pi])$. Then $g(r):=1-\{\sin (\pi r) /(\pi r)\}^{2}$. Note that $|h(r)|$ does not decay exponentially, but rather like the power-law $r^{-2}$ with exponent larger than 1 . The function $h$ is integrable and $\int_{\mathbb{R}} h(r) d r=-1$. Here, $f$ is not integrable but $f^{2}$ is.

The corresponding long-range Hamiltonian of points is the one of the GUE eigenvalues ensemble in random matrix theory

$$
H_{n}\left(\mathbf{x}_{(n)}\right)=-\sum_{1 \leq m_{1}<m_{2} \leq n} \log \left(x_{\left(m_{2}\right)}-x_{\left(m_{1}\right)}\right)+\frac{1}{2 n} \sum_{m=1}^{n} x_{(m)}^{2} .
$$

It exhibits a repulsive Coulombic logarithmic interaction potential at reciprocal temperature $\beta=2$ (see [CL1]).

3./ Let $\rho, \xi>0$ and let $f_{0}(r)=\sqrt{\frac{\rho 2^{1 / \xi}}{\Gamma(1 / \xi)}} \exp \left\{-\left(\frac{r}{2 \xi}+e^{-r}\right)\right\}$ be a Gumbel function on $\mathbb{R}$ (with $\Gamma$ (.) the Euler gamma function). Define $\widetilde{f}_{0}(r):=f_{0}(-r)$ and let $f(r):=f_{0} * \widetilde{f}_{0}(r)$ be the convolution of $f_{0}$ with a symmetrized version of itself. Then, $f$ is symmetric. Further, one can check that $f(r)=\rho\{\cosh (r / 2)\}^{-1 / \xi}$ with $f(0)=\rho$ and $f(r) / f(0) \leq 1$. Function $f$ is integrable and square-integrable.

The Fourier transform of $f_{0}(r)$ is $\sqrt{\frac{\rho 2^{1 / \xi}}{\Gamma(1 / \xi)}} \Gamma\left(\frac{1}{2 \xi}-i \lambda\right)$ so that

$$
\widehat{f}(\lambda):=\frac{\rho 2^{1 / \xi}}{\Gamma(1 / \xi)}\left|\Gamma\left(\frac{1}{2 \xi}-i \lambda\right)\right|^{2} \geq 0,
$$

with $\widehat{f}(0)=\rho 2^{1 / \xi} \frac{\Gamma(1 /(2) \xi)^{2}}{\Gamma(1 / \xi)}$. As a result, $f$ is positive definite. For values of $\rho \in R \subseteq$ $\left(0, \rho_{c}:=:=2^{-1 / \xi} \frac{\Gamma(1 / \xi)}{\Gamma(1 /(2 \xi))^{2}}\right]$ for which $\widehat{f}(0) \leq 1$, the induced determinantal point process is well-defined. The two-point correlation function is $g(r)=1-\{\cosh (r / 2)\}^{-2 / \xi}$ and $h(r)=-\{\cosh (r / 2)\}^{-2 / \xi}$ with $|h(r)| \sim_{r / \infty} 4^{1 / \xi} e^{-r / \xi}$ showing that correlation length is indeed $\xi$. The function $h$ is integrable and $\int_{\mathbb{R}} h(r) d r=-2^{2 / \xi} \frac{\Gamma(1 / \xi)^{2}}{\Gamma(2 / \xi)}$ with $\int_{\mathbb{R}} h(r) d r \sim_{\xi / \infty}-8 \xi$ and $\int_{\mathbb{R}} h(r) d r \sim_{\xi \backslash 0}-(2 \xi)^{-1 / 2}$ diverging at both ends.

4./ Let $f_{1}(r)=\sqrt{\rho} e^{-|r| /(2 \xi)}$ and $f_{2}(r)=\sqrt{\rho} \sin (\pi r) /(\pi r)$ and consider the point-wise product $f(r)=f_{1}(r) \cdot f_{2}(r)$ with $f(0)=\rho$. We have $\widehat{f}(\lambda)=\frac{1}{2 \pi} \widehat{f}_{1} * \widehat{f}_{2}(\lambda)$ where $\widehat{f}_{1}(\lambda)=\frac{\sqrt{\rho} \xi^{-1}}{(2 \xi)^{-2}+\lambda^{2}}$ and $\widehat{f}_{2}(\lambda)=\sqrt{\rho} \cdot \mathbf{1}(\lambda \in[-\pi,+\pi])$. We find $\widehat{f}(\lambda)=\frac{2 \rho \xi}{\pi} \int_{-\pi}^{\pi} \frac{d \lambda^{\prime}}{1+4 \xi^{2}\left(\lambda-\lambda^{\prime}\right)^{2}}=\frac{\rho}{\pi}(\arctan (2 \xi(\lambda+\pi))-\arctan (2 \xi(\lambda-\pi))) \geq 0$. 
The induced determinantal point process is well-defined at least if $2 \rho \arctan (2 \xi \pi) / \pi \leq$ 1 and so $\rho_{c}=\pi /(2 \arctan (2 \xi \pi))$.

The total pair correlation function is $h(r)=-e^{-|r| / \xi} \sin ^{2}(\pi r) /(\pi r)$ with exponential decay and correlation length $\xi$.

5./ Let $f_{1}(r)=f_{2}(r)=\sqrt{\rho} \sin (\pi r) /(\pi r)$ and consider the point-wise product $f(r)=f_{1}(r) \cdot f_{2}(r)=f_{1}(r)^{2}$ with $f(0)=\rho$. We have $\widehat{f}(\lambda)=\frac{1}{2 \pi} \widehat{f}_{1}^{* 2}(\lambda)$ where $\widehat{f}_{1}(\lambda)=\sqrt{\rho} \cdot \mathbf{1}(\lambda \in[-\pi,+\pi])$. With $(x)_{+}:=\max (x, 0)$, we have

$$
\widehat{f}(\lambda)=\rho\left(1-\left|\frac{\lambda}{2 \pi}\right|\right)_{+} \geq 0 .
$$

The induced determinantal point process is well-defined at least if $\rho \leq \rho_{c}=1$. Here, $h(r)=-\{\sin (\pi r) /(\pi r)\}^{4}$. We shall see below that $2 I=\int_{\mathbb{R}} h(r) d r=-2 / 3$.

6./ With $\rho \in R \subseteq\left(0, \rho_{c}:=1\right]$, let $f(r)=\frac{2 \rho}{\pi^{2} r^{2}}\left\{\frac{\sin (\pi r)}{\pi r}-\cos (\pi r)\right\}$ (which is symmetric) satisfying $f(0)=(2 \rho) / 3$. In this case, it can easily be checked that

$$
\widehat{f}(\lambda)=\rho\left(1-\left(\frac{\lambda}{\pi}\right)^{2}\right)_{+},
$$

which has a 'circular' shape. The function $f(r)$ is of positive type. It has infinitely many zeroes, located at points $r$ solving: $\tan (\pi r)=\pi r$. Further, $h(r)=$ $-\left\{\frac{3}{\pi^{2} r^{3}}(\sin (\pi r)-\pi r \cos (\pi r))\right\}^{2}$, satisfying $|h(r)| \sim_{|r| / \infty} C r^{-4}$.

In any case, the density of points $\rho$ should not exceed a critical value $\rho_{c}$ whose value is obtained by imposing $\hat{f}(\lambda) \in[0,1]$. Note that the exact range $R$ of values of $\rho$ for which the DPP is well-defined is a priori included or equal to the interval $\left(0, \rho_{c}\right]$. It could be that $R \subset\left(0, \rho_{c}\right]$; example $\mathbf{6}$./ will show that this is indeed possible.

The 2 -point correlation function $g(r)$ always belongs to $[0,1]$ (so that total correlation $h(r) \in[-1,0]$ is negative); this translates the evidence that translation invariant determinantal (fermion) point processes are always 'repulsive'. Note finally that in Examples 1./, 3./ and 5./, $f(r)$ is doubly positive, that is positive-definite and positive. In Examples 2./, 4./ and 6./, $f(r)$ simply is positive-definite (of positive type).

Number of points; variance growth with volume: Let $x>0$. If $B:=$ $[-x / 2 ; x / 2]$, with $\mathcal{N}_{x}:=\mathcal{N}([-x / 2 ; x / 2])$, if the DPP is translation invariant, we have

$\mathbb{E}\left(\mathcal{N}_{x}\right)=: \eta_{x}=\rho x$ and $\sigma^{2}\left(\mathcal{N}_{x}\right)=: \sigma_{x}^{2}=\rho x+\rho^{2} \int_{[-x / 2 ; x / 2]^{2}}\left(g\left(x_{1}-x_{2}\right)-1\right) d x_{1} d x_{2}$.

An integration by parts yields

$$
\sigma_{x}^{2}=\eta_{x}\left(1+\rho \int_{\mathbb{R}}\left(1-\frac{|r|}{x}\right)_{+} h(r) d r\right),
$$

from which we get: $h(x)=\left(\sigma_{x}^{2}\right)^{\prime \prime} /\left(2 \rho^{2}\right)$, relating the total correlation function $h$ to the second derivative of $\sigma_{x}^{2}$ with respect to $x$. 
If $h$ is integrable, it follows from the last formula that: $\sigma_{x}^{2} / \eta_{x} \rightarrow_{x}{ }_{\infty} 1+$ $\rho \int_{\mathbb{R}} h(r) d r \geq 0$, suggesting that, when $1+\rho \int_{\mathbb{R}} h(r) d r>0$, the variance $\sigma_{x}^{2}$ should also grow like volume $x$.

It may happen that $1+\rho \int_{\mathbb{R}} h(r) d r=0$ so that $\int_{\mathbb{R}} h(r) d r$ attains its lower possible bound $-1 / \rho$. If this is so, $\sigma_{x}^{2} / \eta_{x} \rightarrow_{x} \nearrow_{\infty} 0$ and variance growth is sub-linear.

This will be the case for instance, if $\rho=\rho_{c}=1$ in the second example; at critical density of points, variance grows as $\log x$ (slower than $x$ ); see [CL1] where it is also shown that a Central Limit Theorem for $\mathcal{N}_{x}$ holds. We shall come back to the $\log x$ growth condition later.

For the first example, $1+\rho \int_{\mathbb{R}} h(r) d r \geq 1 / 2\left(=1 / 2\right.$ when $\left.\rho=\rho_{c}=1 /(4 \xi)\right)$ and, although fluctuations are minimal at $\rho=\rho_{c}$, this opportunity is ruled out. The same holds true for the third example, since $1+\rho \int_{\mathbb{R}} h(r) d r \geq 1+\rho_{c} \int_{\mathbb{R}} h(r) d r=$ $1-2^{1 / \xi} \frac{\Gamma(1 / \xi)}{\Gamma(2 / \xi)}\left(\frac{\Gamma(1 / \xi)}{\Gamma(1 /(2 \xi))}\right)^{2}>0$. We shall comment Examples 4./ and 5./ from the point of view of variance growth later.

Point systems for which the variance growth is sub-linear with respect to volume are called super-homogeneous (see [GJL]). If in addition there is a constant $C$ such that $\sigma_{x}^{2} \leq C$ so that the variance saturates, the point process is said to be hyper-uniform (see [TS]). Hyper-uniform PP constitute a proper sub-class of superhomogeneous PP.

The simplest example of a translation invariant hyper-uniform PP is as follows (see [GT], page 041105): Take the lattice with distance $D>0$ between consecutive points. In each cell of length $D$ put a point uniformly at random, independently for each cell and consider the random set of all these points. Although random, this process inherits part of the determinism of the underlying lattice. The obtained jittered point process is translation invariant and hyper-uniform. Similar behavior are observed for quasi-crystals $([\mathrm{GO}])$. Another far less intuitive example is the socalled G-process defined in [GLS] which may either be viewed as the output of a $\mathrm{D} / \mathrm{M} / 1$-queue or as a self-correcting (or stress release) process, see also [DVJ], pages 244-245.

Such 'glassy' hyper-uniform systems present some long-range order and are stronglycorrelated. They are believed to be of some interest in the Harrison-Zeldovich theory of cosmology. See [GJL], for a 3-dimensional version of the hyper-uniformity concept.

Determinantal super-homogeneous systems are to be searched in systems for which the spectral density $\widehat{f}(\lambda)$ has compact support (as in Example 2./): they do not possess infinite wave-length fluctuations. Example 2./ is super-homogeneous with logarithmic number variance growth with length $x$. Are there translation invariant DPP which are hyper-uniform? Before addressing this question, let us investigate an important class of translation invariant PP which cannot be super-homogeneous, as they are 'too' random, being 'essentially Poissonian'.

\subsection{Renewal point processes gas}

Construction, definitions and properties: Consider a 'delayed' renewal point process (RPP) on the real line, defined as follows: starting from the origin 0 , which 
is not a point of the point process, assume the distance (delay) $\Delta_{0}$ till the first point met to the right is random with pdf $f_{0}$. Starting from this first point, consecutive points to the right are at mutual random distances $\left(\Delta_{m} ; m \geq 1\right)$ of one another, where the $\Delta_{m}$ are iid with common density $f$. Assume $\mathbb{E}\left(\Delta_{1}\right)=\mu:=1 / \rho<\infty$. Assume also that $\Delta_{0}$ is independent of $\left(\Delta_{m} ; m \geq 1\right)$. Let $\Delta_{0}^{\prime} \stackrel{d}{=} \Delta_{0}$ be the distance to the origin of the first point met to the left of 0 . For consecutive points, proceed similarly and independently to the left with $\Delta_{-m} \stackrel{d}{=} \Delta_{m}, m \geq 1$ representing the mutual distances between consecutive points to the left. We are left with a point process $\left(X_{(n)} ; n \in \mathbb{Z} \backslash\{0\}\right)$ on $\mathbb{R}$ :

$$
X_{(n)}=\Delta_{0}+\sum_{m=1}^{n-1} \Delta_{m} \text { and } X_{(-n)}=-\Delta_{0}^{\prime}-\sum_{m=1}^{n-1} \Delta_{-m}, n \geq 1 .
$$

Let $\Lambda_{0}(x):=\mathbb{E}\left(N_{x}\right)$ be the expected number of points in the interval $[-x / 2, x / 2]$ centered at the origin. $\Lambda_{0}(x)$ is absolutely continuous with respect to Lebesgue measure with density of points $\lambda_{0}(x)$ at $x$ satisfying $\lambda_{0}(x)=\lambda_{0}(-x)$ and, by Blackwell Renewal Theorem, $\lambda_{0}(x) \rightarrow \rho$ as $x \nearrow \infty$. Let $\widehat{\lambda}_{0}(s)=\int_{0}^{\infty} e^{-s x} \lambda_{0}(x) d x$ and $\left(\widehat{f}_{0}(s) ; \widehat{f}(s)\right)$ denote respectively the Laplace-Stieltjes transforms of $\lambda_{0}(x), f_{0}(x)$ and $f(x)$ on the half-line. Then, usual the renewal structure of the point process,

$$
\widehat{\lambda}_{0}(s)=\frac{\widehat{f}_{0}(s)}{1-\widehat{f}(s)} .
$$

For the specific choice of the joint pdf of $\left(\Delta_{0}^{\prime}, \Delta_{0}\right): f_{\Delta_{0}^{\prime}, \Delta_{0}}\left(x^{\prime}, x\right)=\rho f\left(x^{\prime}+x\right)$, the marginal pdf $f_{0}$ of $\Delta_{0}$ (and $\Delta_{0}^{\prime}$ ) reads:

$$
f_{0}(x)=\rho\left(1-\int_{0}^{x} f(z) d z\right) .
$$

We therefore get $\widehat{f}_{0}(s)=\frac{\rho}{s}(1-\widehat{f}(s))$ so that $\widehat{\lambda}_{0}(s)=\rho / s$; this shows that $\lambda_{0}(x)=$ $\rho$ is constant. Such specific delayed renewal point processes are called translation invariant: For each point $x \in \mathbb{R}$, the joint law of the distances of the first point met to the left and to the right of $x$ coincides with the one of $\left(\Delta_{0}^{\prime}, \Delta_{0}\right)$ at the origin (see $[\mathrm{SVH}])$.

If $\left(X_{(n)} ; n \in \mathbb{Z} \backslash\{0\}\right)$ denotes the ordered random positions of points on the line of a translation invariant $\operatorname{RPP},\left(X_{(n)} ; n \in \mathbb{Z} \backslash\{0\}\right)$ is Markovian in that for each $n \in \mathbb{Z} \backslash\{0\}$

$$
\begin{aligned}
& \mathbb{P}\left(\left\{X_{(m)} \in d x_{(m)}\right\}_{m \geq n} \mid\left\{X_{(m)}=x_{(m)}\right\}_{m<n}\right) \\
= & \mathbb{P}\left(\left\{X_{(m)} \in d x_{(m)}\right\}_{m \geq n} \mid X_{(n-1)}=x_{(n-1)}\right) .
\end{aligned}
$$

In particular,

$$
\mathbb{P}\left(X_{(n)} \in d x_{(n)} \mid\left\{X_{(m)}=x_{(m)}\right\}_{m<n}\right) / d x_{(n)}=f\left(x_{(n)}-x_{(n-1)}\right) .
$$


Consider now the statistical features of the underlying 'pure' renewal point process. Define $\Lambda(x)$ to be the expected number of points in the interval $[-x / 2, x / 2]$ centered at the origin when the origin now is a point of the point process and when the mutual random distances $\left(\Delta_{m} ; m \in \mathbb{Z} \backslash\{0\}\right)$ between consecutive points to the right (and then independently to the left), are iid with common density $f . \Lambda(x)$ is absolutely continuous with respect to Lebesgue measure with density of points $\lambda(x)$, with $\lambda(x) \rightarrow \rho$ as $x \nearrow \infty$. Clearly, with $\widehat{\lambda}(s)=\int_{0}^{\infty} e^{-s x} \lambda(x) d x$,

$$
\widehat{\lambda}(s)=\frac{\widehat{f}(s)}{1-\widehat{f}(s)} .
$$

With $x_{(1)} \leq . . \leq x_{(n)}$, the $n$-point correlation functions of the delayed renewal point process are given by (see $[\mathrm{DVJ}])$

$$
\rho_{n}\left(\mathbf{x}_{(n)}\right)=\lambda_{0}\left(x_{(1)}\right) \prod_{m=2}^{n} \lambda\left(x_{(m)}-x_{(m-1)}\right) .
$$

When the process is translation invariant $\rho_{n}\left(\mathbf{x}_{(n)}\right)=\rho \prod_{m=2}^{n} \lambda\left(x_{(m)}-x_{(m-1)}\right)$; in particular $\rho_{1}\left(\mathbf{x}_{(1)}\right)=\rho$ and $\rho_{2}\left(\mathbf{x}_{(2)}\right)=\rho^{2} g\left(x_{(2)}-x_{(1)}\right)$ where $g(r):=\lambda(r) / \rho \rightarrow 1$ as $r \nearrow \infty$. The Janossy densities are given by (see [DVJ])

$$
v_{n}\left(\mathbf{x}_{(n)}\right)=\rho \prod_{m=2}^{n} f\left(x_{(m)}-x_{(m-1)}\right) .
$$

If the variance $\sigma^{2}$ of $\Delta_{1}$ is finite, if $I(r):=\int_{0}^{r}(g(x)-1) d x$, we have

$$
\widehat{I}(s):=\int_{0}^{\infty} e^{-s r} I(r) d r \sim_{s \searrow 0} \frac{-1}{2 \mu s}\left(\mu^{2}-\sigma^{2}\right)
$$

showing, by Tauberian theorem, that $I(r) \rightarrow I:=\frac{-1}{2 \mu}\left(\mu^{2}-\sigma^{2}\right)$ as $r \nearrow \infty$. If $\mu \geq \sigma$ (respectively $\mu<\sigma$ ), $I \leq 0$ (respectively $I>0$ ). Therefore, for translation invariant renewal point processes with finite spacings variance, the ratio of the variance of $\mathcal{N}_{x}$ to its mean satisfies

$$
\sigma_{x}^{2} / \eta_{x} \rightarrow_{x}{ }_{\infty} 1-\frac{1}{\mu^{2}}\left(\mu^{2}-\sigma^{2}\right)=\frac{\sigma^{2}}{\mu^{2}}>0 .
$$

Such processes can never be super-homogeneous: large scale fluctuations are identical to the ones of the Poisson point process as they grow like volume $x$. However, if $\mu>\sigma$ (respectively $\mu<\sigma)$, the normalized variance $\frac{\sigma^{2}}{\mu^{2}}<1$ (respectively $\frac{\sigma^{2}}{\mu^{2}}>1$ ) and therefore fluctuations are smaller (respectively larger) than the ones expected for the Poisson process for which $\frac{\sigma^{2}}{\mu^{2}}=1$. This can be used to distinguish between super- (respectively sub-) Poissonian point processes.

Examples from Gibbs measures: Let $U(x)$ stand for some interaction potential, with $U(x) \searrow 0$ as $x \nearrow \infty$. If $U(x)>0$ when $x>0$, potential $U$ is purely repulsive whereas if $U(x)<0$ when $x<0$, potential $U$ is purely attracting. If none of these conditions is fulfilled, one can always define the repulsive (attracting) domains of $U$ to be $\{x>0: U(x)>0\}$ (respectively, $\{x>0: U(x)<0\}$ ). In the set 
$\{x>0: U(x)=0\}, U$ is 'neutral'. If $U$ is attracting in a neighborhood of $x=0$, assume $U(x)>\delta \log x$ where $\delta \in(0,1 / \beta)$, locally.

Let $p$ stand for thermodynamical 'pressure', $\beta$ for reciprocal 'temperature', $\beta>0$. Assume the pdf of $\Delta_{1}$ is given by

$$
f(x)=\frac{1}{Z_{\beta}(p)} e^{-\beta(p x+U(x))}, x>0,
$$

where

$$
Z_{\beta}(p)=\int_{0}^{\infty} e^{-\beta(p x+U(x))} d x .
$$

Under our assumptions on $U, \mathbb{E}\left(\Delta_{1}\right)=1 / \rho<\infty$ and, with $F_{\beta}(p):=-\frac{1}{\beta} \log Z_{\beta}(p)$, the equation of state reads

$$
\frac{1}{\rho}=\partial_{p} F_{\beta}(p) \text {. }
$$

From the expression of the Janossy densities in this case, the Hamiltonian of such an $n$-particle system at $\mathbf{x}_{(n)}$ only exhibit nearest-neighbor interactions with

$$
H_{n}\left(\mathbf{x}_{(n)}\right)=\sum_{m=2}^{n} U\left(x_{(m)}-x_{(m-1)}\right)+p\left(x_{(n)}-x_{(1)}\right) .
$$

Because $\widehat{f}(s)=\frac{Z_{\beta}(p+s / \beta)}{Z_{\beta}(p)}$, the intensity function $\lambda$ appearing in the correlation functions is characterized by

$$
\widehat{\lambda}(s)=\left(\frac{Z_{\beta}(p)}{Z_{\beta}(p+s / \beta)}-1\right)^{-1}=\frac{Z_{\beta}(p+s / \beta)}{Z_{\beta}(p)-Z_{\beta}(p+s / \beta)} .
$$

When $s$ is small, $\hat{\lambda}(s) \sim \rho / s$ in accordance, by Tauberian theorem, with $\lambda(r) \rightarrow \rho$ as $r \nearrow \infty$.

Let us now investigate some Examples.

$\star$ Assume first $U(x)=0$, for all $x>0$ (no interaction). Then $Z_{\beta}(p)=\frac{1}{\beta p}$; the equation of state reads $\frac{p}{\rho}=\beta^{-1}$. In this case, $\widehat{\lambda}(s)=(\beta p) / s$ so that $g(r)=$ $\lambda(r) / \rho=\mathbf{1}(r>0)$ is constant. The underlying random point process is the interaction free Poisson process with intensity $\rho$. Here $I=0$ and $\sigma_{x}^{2} / \eta_{x} \rightarrow_{x}{ }_{\infty} 1$ (actually, $\sigma_{x}^{2} / \eta_{x}=1$, for all $\left.x\right)$.

* Take for instance the hard-core model for which $U(x)=\infty \cdot \mathbf{1}(x<D)+0$. $\mathbf{1}(x \geq D)$. Here $Z_{\beta}(p)=\frac{1}{\beta p} e^{-\beta p D}$ and with $\rho<\rho_{c}=D^{-1}$, the equation of state reads $p\left(\frac{1}{\rho}-D\right)=\beta^{-1}$. Here, $\widehat{\lambda}(s)=\left((1+s /(\beta p)) e^{s D}-1\right)^{-1}$. Using the equation of state and the identity

$$
\frac{1}{\Gamma(a)} \int_{0}^{\infty}\left(r-r_{0}\right)_{+}^{a-1} e^{-b\left(r-r_{0}\right)} e^{-s r} d r=(b+s)^{-a} e^{-s r_{0}}, \text { with } a, b, r_{0}>0
$$

a series expansion of $\widehat{\lambda}(s)$ leads by Laplace inversion to

$$
g(r)=\lambda(r) / \rho=\frac{1}{\rho} \sum_{m \geq 1} \frac{1}{\Gamma(m)}(1 / \rho-D)^{-m} e^{-\frac{(r-m D)}{1 / \rho-D}}(r-m D)_{+}^{m-1}, r>0
$$




$$
=\left(D+\frac{1}{\beta p}\right) \sum_{m \geq 1} \frac{1}{\Gamma(m)}(\beta p)^{m} e^{-\beta p(r-m D)}(r-m D)_{+}^{m-1} .
$$

From the expression of $g(r)$ we have $g(r)=0$ if $r<D$ and $g(D)=(1-\rho D)^{-1}=$ $(\beta p) / \rho>1$. This last expression constitutes an old result from equilibrium Statistical Physics (see for example (A17) of [MS] page 3155). From the above expression, one can prove that the limit $\frac{1}{\xi}:=\liminf \operatorname{in}_{r \uparrow \infty}-\frac{1}{r} \log |h(r)|$ exists, with $\xi$ therefore interpreting as the correlation length of the $2-$ point correlation function.

It can be checked that

$$
I:=\int_{0}^{\infty} h(r) d r=-\frac{1}{2 \mu}\left(\mu^{2}-\sigma^{2}\right)=-\{D(2+\beta p D)\} /\{2(1+\beta p D)\}<0
$$

and

$$
\sigma_{x}^{2} / \eta_{x} \rightarrow_{x>\infty}(1+\beta p D)^{-2}>0 .
$$

Since $(1+\beta p D)^{-2}<1$, the hard-core model is super-Poissonian as $\mu>\sigma$.

* Take $U(x)=-u \log \left(1-e^{-x / D}\right)$ where $u, D>0, D$ fixing the length scale. Then $f(x)=\frac{1}{Z_{\beta}(p)} e^{-\beta p x}\left(1-e^{-x / D}\right)^{\beta u}$ where

$$
\begin{gathered}
Z_{\beta}(p)=\int_{0}^{\infty} e^{-\beta p x}\left(1-e^{-x / D}\right)^{\beta u} d x \\
=D \int_{0}^{1} z^{\beta p D-1}(1-z)^{\beta u} d z=D \frac{\Gamma(\beta p D) \Gamma(\beta u+1)}{\Gamma(\beta p D+\beta u+1)} .
\end{gathered}
$$

If $u<0$, potential $U$ is purely attracting, with $U(x) \sim_{x \backslash 0}-u \log x$ and for the process to be well-defined, $u$ needs to be larger than $-\beta^{-1}$. If $u>0$, it is purely repulsive. Let us consider a special case of importance.

Specifying $u=1 / \beta$, potential $U$ is purely repulsive and the pdf $f(x)$ is a linear combination of two exponentials. Further,

$$
Z_{\beta}(p)=\frac{1}{\beta p(\beta p D+1)} .
$$

The equation of state reads

$$
\frac{p}{\rho}=\beta^{-1}+\frac{p D}{\beta p D+1} .
$$

In terms of the correlation length $\xi=\frac{D}{2 \beta p D+1}$, it can easily be checked from Laplace inversion of $\widehat{\lambda}(s)$ that $g(r)=1-e^{-r / \xi}, r>0$. Clearly, $I=-\xi=-\frac{1}{2 \mu}\left(\mu^{2}-\sigma^{2}\right)$ where $\mu=\rho^{-1}$ and $\sigma^{2}=(p \beta)^{-2}+D^{2} /(\beta p D+1)^{2}$.

Further,

$$
\frac{1}{\rho \xi}=4+\frac{1}{(\beta p D+1) \beta p D}>4,
$$

showing that $4 \rho \xi<1$ (or $\rho<\rho_{c}:=1 /(4 \xi)$ ). When length scale $D \nearrow \infty, 4 \rho \xi \searrow 1$ and the limiting pdf $f(x)=(\beta p)^{2} x e^{-\beta p x}$ still is a well-defined density on $(0, \infty)$. The limiting critical equation of state reads: $\frac{p}{\rho}=2 \beta^{-1}$ whereas correlation length $\xi$ 
tends to $(2 \beta p)^{-1}$. Thus, the full range of admissible parameters $(\rho, \xi)$ is $4 \rho \xi \leq 1$ (or $\left.\rho \leq \rho_{c}:=1 /(4 \xi)\right)$.

Comparing with the first example 1./ of translation invariant determinantal point process, this remarkable renewal process is also determinantal as both processes share the same $n$-point correlations. Actually, it is the only one (see [S] and $[\mathrm{M}]$ ).

The above limiting ( critical) process for large $D$ suggests that the full model (14) should be exactly solvable even if $u \neq 1 / \beta$, when $\rho$ approaches its largest admissible value. Suppose indeed $u=(\alpha-1) / \beta$ where $\alpha>0$. If $\alpha \in(0,1)$ potential $U(x)=$ $-u \log \left(1-e^{-x / D}\right)$ is purely attracting, neutral if $\alpha=1$ whereas $\alpha>1$ is a purely repulsive case, with $\alpha=2$ coinciding with the previous case study. Using the large $D$ expansion of the Euler gamma function appearing in the expression of $Z_{\beta}(p)$, we get

$$
Z_{\beta}(p) \sim_{D \nearrow \infty} D^{-(\alpha-1)} \frac{\Gamma(\alpha)}{(\beta p)^{\alpha}}
$$

corresponding to the critical limiting equation of state $\frac{p}{\rho}=\alpha \beta^{-1}$. It corresponds to a large $D$ limiting density of type gamma $(\alpha, \beta p)$ for $\Delta_{1}$

$$
f(x)=\frac{(\beta p)^{\alpha}}{\Gamma(\alpha)} x^{\alpha-1} e^{-\beta p x}
$$

with $\widehat{f}(s)=(1+s /(\alpha \rho))^{-\alpha}$. We have $\mu=\rho^{-1}$ and $\sigma^{2}=1 /\left(\alpha \rho^{2}\right)$. Thus

$$
\widehat{\lambda}(s)=\frac{\widehat{f}(s)}{1-\widehat{f}(s)}=\sum_{m \geq 1}(1+s /(\alpha \rho))^{-m \alpha}
$$

showing by Laplace inversion that $g(r)$ takes the exact form

$$
g(r)=\frac{e^{-\beta p r}}{\rho r} \sum_{m \geq 1} \frac{(\beta p r)^{m \alpha}}{\Gamma(m \alpha)}=\frac{\alpha(\beta p)^{\alpha}}{\rho} r^{\alpha-1} e^{-\beta p r} d_{\alpha}\left((\beta p r)^{\alpha}\right), r>0
$$

Here $d_{\alpha}(x):=e_{\alpha}^{\prime}(x)$ is the derivative with respect to $x$ of $e_{\alpha}(x):=\sum_{m \geq 0} \frac{x^{m}}{\Gamma(m \alpha+1)}$, the Mittag-Leffler exponential function. We may check that this novel correlation function is consistent with what we already know:

When $\alpha=1, e_{1}(x)=\exp (x)$ and $g(r)=1, r>0$. The RPP is a Poisson point process with intensity $\rho=p \beta$.

When $\alpha=2, e_{2}(x)=\cosh (\sqrt{x}), d_{2}(x)=\frac{1}{2 \sqrt{x}} \sinh (\sqrt{x})$ and $g(r)=1-e^{-r / \xi}$, where $\xi=(2 \beta p)^{-1}, r>0$.

When $\alpha=1 / 2, e_{2}(x)=e^{x^{2}} \operatorname{erf} c(-x)$ and $d_{2}(x)=2\left(x e_{2}(x)+1 / \sqrt{\pi}\right)$ where $\operatorname{erf} c(x):=2 / \sqrt{\pi} \int_{x}^{\infty} e^{-z^{2}} d z$. We have $g(r) \nearrow \infty$ as $r \searrow 0$ since $g(r)$ is of order $r^{-1 / 2}$.

One can check that $I=\int_{0}^{\infty} h(r) d r=(1-\alpha) /(2 \beta p)=\left(1-\alpha^{-1}\right) /(2 \rho)$. This quantity is positive when $\alpha<1$. Further, $\sigma_{x}^{2} / \eta_{x} \rightarrow_{x / \infty} \alpha^{-1}$. The case $\alpha>1$ (respectively $\alpha<1$ ) corresponds to a super-(respectively sub-) Poissonian case. 
* Even for simple models, the correlation function can be hard to evaluate. Take for example the purely repulsive potential $U$ defined by $U(x)=u \cdot(x / D)^{-1}$, where $u, D$ are positive constants. $U$ is monotone decreasing with $U(x) \rightarrow \infty$ as $x \searrow 0$. In this particular case, the mutual distance between points has generalized inverse Gaussian density, namely

$$
f(x)=\frac{1}{\left(\frac{u D}{p}\right)^{1 / 2} K(\beta \sqrt{u p D})} \exp \{-\beta(p x+u D / x)\}, x>0
$$

where $K(z)=\int_{0}^{\infty} \exp \{-z(x+1 / x)\} d x$ is a modified Bessel function of the third kind. Thus, $Z_{\beta}(p)=\left(\frac{u D}{p}\right)^{1 / 2} K(\beta \sqrt{u p D})$ and

$$
\widehat{f}(s)=\left(\frac{p}{p+s / \beta}\right)^{1 / 2} K(\beta \sqrt{(p+s / \beta) u D}) / K(\beta \sqrt{u p D}) .
$$

The Laplace inverse of $\widehat{\lambda}(s)=\widehat{f}(s)(1-\widehat{f}(s))^{-1}$ is not known.

Even worse, take the Lennard-Jones model, with $a, b>0, U(x)=a x^{-6}-b x^{-3}$ with $U(x) \sim a x^{-6} \rightarrow \infty$ as $x \searrow 0$ and $U(x) \sim-b x^{-3} \rightarrow 0$ as $x \nearrow \infty$. Potential $U$ has a minimum at $x_{*}=(2 a / b)^{1 / 3}$ with $U_{*}:=U\left(x_{*}\right)<0$. For $x>(a / b)^{1 / 3}$, potential $U$ is attracting. In that case, there is even no simple expression of the normalizing constant $Z_{\beta}(p)$ so that both $\widehat{f}(s)$ and $\widehat{\lambda}(s)$ are out of reach.

\section{Super- and sub-homogeneous point processes}

With $\varphi \in L^{1}(\mathbb{R}) \cap L^{2}(\mathbb{R})$, consider the additive functional $\psi:=\sum_{N=1}^{\infty} \varphi\left(X_{N}\right)$ of some translation invariant random $\mathrm{PP}$ with infinitely many points $\left(X_{N} ; N \geq 1\right)$. Its variance is given in terms of total correlation function $h$ by

$$
\sigma_{\psi}^{2}=\int_{\mathbb{R}} \int_{\mathbb{R}} \varphi\left(x_{1}\right) \varphi\left(x_{2}\right)\left(\rho \delta_{x_{2}-x_{1}}+\rho^{2} h\left(x_{2}-x_{1}\right)\right) d x_{1} d x_{2} .
$$

In Fourier space, it has the spectral representation

$$
\sigma_{\psi}^{2}=\frac{1}{2 \pi} \int_{\mathbb{R}}|\widehat{\varphi}(\lambda)|^{2} \widehat{S}(\lambda) d \lambda
$$

where $\widehat{\varphi}(\lambda)=\int_{\mathbb{R}} e^{i \lambda x} \varphi(x) d x$ is the Fourier transform of $\varphi$ and $\widehat{S}(\lambda)$ is the Bartlett power-spectrum density

$$
\widehat{S}(\lambda)=\rho+\rho^{2} \widehat{h}(\lambda)=: \rho \widehat{s}(\lambda) \geq 0 .
$$

Here $\widehat{s}(\lambda)=1+\rho \widehat{h}(\lambda)$ is the structure factor and $\widehat{h}(\lambda)=\int_{\mathbb{R}} e^{i \lambda r} h(r) d r$, the Fourier transform of the total correlation function $h(r)$. The function $\rho^{2} \widehat{h}(\lambda)$ is also called the Cramér power-spectrum. When $\varphi(X)=\mathbf{1}\left(X \in\left[-\frac{x}{2}, \frac{x}{2}\right]\right)$ is the indicator of the window interval $\left[-\frac{x}{2}, \frac{x}{2}\right], \psi:=\sum_{N=1}^{\infty} \mathbf{1}\left(X_{N} \in\left[-\frac{x}{2}, \frac{x}{2}\right]\right)$ counts the number of points in this interval and so $\psi$ is $\mathcal{N}_{x}$ and $\sigma_{\psi}^{2}$ coincides with $\sigma_{x}^{2}$, the number of points variance in the window. In this case, $\widehat{\varphi}(\lambda)=x \frac{\sin \left(\lambda \frac{x}{2}\right)}{\lambda \frac{x}{2}}$ and

$$
\sigma_{x}^{2}=\frac{x^{2}}{2 \pi} \int_{\mathbb{R}}\left\{\frac{\sin \left(\lambda \frac{x}{2}\right)}{\lambda \frac{x}{2}}\right\}^{2} \widehat{S}(\lambda) d \lambda
$$


After the change of variables $u=\lambda \frac{x}{2}$, this is also

$$
\sigma_{x}^{2}=\frac{\rho x}{\pi} \int_{\mathbb{R}}\left\{\frac{\sin (u)}{u}\right\}^{2} \widehat{s}\left(\frac{2 u}{x}\right) d u .
$$

Large $x$ behavior of $\sigma_{x}^{2}$ is governed by the behavior of the structure factor $\widehat{s}($.$) in$ a neighborhood of zero. Recalling $\eta_{x}=\rho x$ is the expected number of points in the interval of length $x$, we also have

$$
\frac{\sigma_{x}^{2}}{\eta_{x}}=\frac{2}{\pi} \int_{0}^{\infty}\left\{\frac{\sin (u)}{u}\right\}^{2} \widehat{s}\left(\frac{2 u}{x}\right) d u
$$

and large $x$ behavior of $\sigma_{x}^{2} / \eta_{x}$ is also controlled by the behavior of $\widehat{s}($.$) in a neigh-$ borhood of zero.

Super-homogeneous point processes with sub-linear variance growth with volume $x$ may be characterized by $\widehat{s}(\lambda) \sim_{|\lambda| \backslash 0} C|\lambda|^{\gamma} e^{-|\lambda| /\left(2 \lambda_{*}\right)}$ where $C, \gamma$ and $\lambda_{*}$ are positive constants. Adapting the considerations of [GJL] (who rather consider the 3-dimensional case), to our 1-dimensional case, we indeed have:

- If $\gamma \in(0,1)$, with $K$ some finite positive constant

$$
\sigma_{x}^{2} \sim_{x \succ \infty} C \frac{2^{\gamma+1} \rho x^{1-\gamma}}{\pi} \int_{0}^{\infty}\{\sin (u)\}^{2} u^{\gamma-2} d u<K \rho x^{1-\gamma},
$$

so that $\frac{\sigma_{x}^{2}}{\eta_{x}} \sim K x^{-\gamma} \rightarrow 0$ (as $\left.x \nearrow \infty\right)$. The number variance grows algebraically but slower than interval length $x$.

- If $\gamma>1$

$$
\sigma_{x}^{2} \sim_{x \nearrow \infty} C \frac{2^{\gamma+1} \rho x^{1-\gamma}}{\pi} \int_{0}^{\infty}\{\sin (u)\}^{2} u^{\gamma-2} e^{-u /\left(\lambda_{*} x\right)} d u .
$$

Since $\int_{0}^{\infty}\{\sin (u)\}^{2} u^{\gamma-2} e^{-u /\left(\lambda_{*} x\right)} d u<\int_{0}^{\infty} u^{\gamma-2} e^{-u /\left(\lambda_{*} x\right)} d u=\Gamma(\gamma-1)\left(\lambda_{*} x\right)^{\gamma-1}$, as $x \nearrow \infty$

$$
\sigma_{x}^{2}<C \rho \frac{2^{\gamma+1}}{\pi} \lambda_{*}^{\gamma-1} \Gamma(\gamma-1)=K^{\prime}
$$

and the number variance saturates. Recall that a super-homogeneous PP with bounded variability is called hyper-uniform.

- If $\gamma=1$, with $K^{\prime \prime}$ some finite positive constant

$$
\sigma_{x}^{2} \sim_{x / \infty} K^{\prime \prime} \log x
$$

with logarithmic variance growth. Indeed, when $\gamma$ tends to 1 from above, slowly enough, from the previous estimates,

$$
\Gamma(\gamma-1)\left(\lambda_{*} x\right)^{\gamma-1} \sim \frac{\lambda_{*}^{\gamma-1}}{\gamma-1}(1+(\gamma-1) \log x) \sim \log x,
$$

showing that $K^{\prime \prime}=\frac{4 \rho C}{\pi}$. 


\section{Additional remarks:}

(i) Assume the point system is super-homogeneous. Defining the direct correlation function $c(r)$ from the 'renewal' equation

$$
h(r)=c(r)+\rho \int_{\mathbb{R}} h\left(r-r^{\prime}\right) c\left(r^{\prime}\right) d r^{\prime}
$$

and taking the Fourier transform, with $\widehat{c}(\lambda)=\int_{\mathbb{R}} e^{i \lambda r} c(r) d r$, we get:

$$
\widehat{c}(\lambda)=\frac{\widehat{h}(\lambda)}{1+\rho \widehat{h}(\lambda)}=\frac{\widehat{s}(\lambda)-\rho}{\rho \widehat{s}(\lambda)} .
$$

Since $\widehat{s}(\lambda) \sim_{|\lambda| \searrow_{0}} C|\lambda|^{\gamma}$ where $\gamma>0, \widehat{c}(\lambda) \sim_{|\lambda| \searrow_{0}}-|\lambda|^{-\gamma}$ diverges in a neighborhood of wave-length $\lambda=0$. For super-homogeneous systems, the direct correlation function is long-ranged.

(ii) It may happen that $\widehat{s}(0)=C$ for some positive constant $C$. For example, this was the case for finite variance renewal point processes with $\widehat{s}(0)=1+2 \rho I=\frac{\sigma^{2}}{\mu^{2}}>0$. In these cases, $\sigma_{x}^{2} / \eta_{x} \rightarrow_{x}{ }_{\infty} C$ and variance grows like volume $x$, just like for Poisson PP. RPP systems with finite spacings variance possess a bona fide correlation length $\xi$.

(iii) Sub-homogeneous or critical systems: For some PP, it may finally happen that $\widehat{s}(\lambda) \sim_{|\lambda| \backslash 0} C|\lambda|^{-\delta}$ for some $\delta \in(0,1)$ and $C>0$. The structure factor diverges in a neighborhood of wave-length $\lambda=0$. If this is so, as $x \nearrow \infty$

$$
\sigma_{x}^{2}=\frac{2 \rho x}{\pi} \int_{0}^{\infty}\left\{\frac{\sin (u)}{u}\right\}^{2} \widehat{s}\left(\frac{2 u}{x}\right) d u \sim \kappa x^{1+\delta}
$$

where $\kappa=\frac{2^{1-\delta} \rho C}{\pi} \int_{0}^{\infty} \sin ^{2}(u) u^{-(\delta+2)} d u<\infty$ is well-behaved because $\delta \in(0,1)$. In this case, the variance grows algebraically and faster than volume $x$ (but still slower than volume squared $x^{2}$ ). This class of PP corresponds to the one of 'critical' systems for which the total 2-point correlation function $h$ itself is long-ranged with power-law decay with exponent in $(0,1)$. We shall give an example of a critical PP in the sequel which is in the class of permanental (boson) PP. In the process, we shall also see that it is possible to have $\widehat{s}(\lambda) \sim|\lambda|\rangle_{0}-C \log |\lambda|$ with a logarithmic divergence of $\widehat{s}(\lambda)$ at $\lambda=0$. In this case, $\sigma_{x}^{2}$ still grows faster than $x$ but with a logarithmic correction. Critical PP for which $\sigma_{x}^{2} / \eta_{x} \rightarrow_{x} \nearrow_{\infty}+\infty$ may as well be called 'sub-homogeneous': they exhibit large disorder.

An immediate first example of such sub-homogeneous point processes is an RPP with infinite spacings variance $\sigma^{2}$ and finite mean $\mu$. More precisely, let $F(x)=$ $\int_{0}^{x} f(z) d z$ be the cumulative probability distribution of spacing $\Delta_{1}$ in the above construction of a RPP. It may easily be shown that, with $\delta \in(0,1)$, if $1-F(x) \sim x / \infty$ $x^{-(2-\delta)} L(x)$ for some slowly varying function $L$, we have: $\left(\mu=1 / \rho<\infty ; \sigma^{2}=\infty\right)$ and

$$
\frac{\sigma_{x}^{2}}{\eta_{x}} \sim \frac{2 \rho^{2} x^{\delta} L(x)}{\delta\left(1-\delta^{2}\right)} \rightarrow \infty, \text { as } x \nearrow \infty
$$


In this case, one can indeed check that, with $\widehat{g}(s)$ and $\widehat{f}(s)$ the respective Laplace transforms of $g(r)$ and $f(x)$ satisfying $\rho \widehat{g}(s)=\widehat{f}(s) /(1-\widehat{f}(s))$, Tauberian theorem gives $\widehat{f}(s) \sim_{s \backslash 0} 1-\mu s+C s^{2-\delta} L(1 / s)$ so that:

$$
\widehat{s}(\lambda):=1+\rho(\widehat{g}(|\lambda|)-1 /|\lambda|) \sim_{|\lambda| \backslash 0} C|\lambda|^{-\delta} L(1 /|\lambda|) .
$$

See $[D V]$ for additional details. These authors call this property the long-range count dependence; parameter $\delta$ is related to the Hurst index through $H=(\delta+1) / 2 \epsilon$ $(1 / 2,1)$; see [DV] and [H2]. The origin of 'Hurstiness' clearly is to be found in the heavy-tailed character of consecutive point spacings distribution. It is also shown in [DV] that this long-range property is shared by the output of some suitably chosen queueing processes.

We now focus on the class of DPP and consider the conditions under which they can be super-homogeneous: In this specific DPP case, we have $\widehat{s}(\lambda)=1+\rho \widehat{h}(\lambda)$ where

$$
\widehat{h}(\lambda)=\frac{-1}{f(0)^{2}} \int_{\mathbb{R}} e^{i \lambda r} f(r)^{2} d r=\frac{-1}{2 \pi f(0)^{2}} \widehat{f}^{* 2}(\lambda)
$$

is given in terms of $\widehat{f}(\lambda)=\int_{\mathbb{R}} e^{i \lambda r} f(r) d r$, the Fourier transform of $f(r)$ which is essentially definite-positive.

\section{Illustrative examples:}

1./ With $\rho, \xi>0$, we had $f(r)=\rho e^{-|r| /(2 \xi)}$, with $\widehat{f}(\lambda)=\frac{\rho \xi^{-1}}{(2 \xi)^{-2}+\lambda^{2}}$. Thus $h(r)=-e^{-|r| / \xi}$ and using stability of Cauchy densities under convolution

$$
\begin{aligned}
& \widehat{h}(\lambda)=\frac{-8 \xi^{2}}{\pi}\left(\frac{1}{1+(2 \xi \lambda)^{2}}\right)^{* 2}=-2 \xi\left(\frac{1}{1+(\xi \lambda)^{2}}\right) \\
& \widehat{s}(\lambda)=1-\frac{2 \xi \rho}{1+(\xi \lambda)^{2}} .
\end{aligned}
$$

As announced, $\widehat{h}(0)=2 I=\int_{\mathbb{R}} h(r) d r=-2 \xi$. Here $\widehat{s}(0)=C$ where $C=1-2 \xi \rho \geq$ $1 / 2$. This determinantal point process is neither super-homogeneous nor 'critical', but rather has a large scale Poisson-like behavior. Recall this determinantal model may also be viewed as a nearest-neighbor renewal point process. Its range $R$ is exactly $(0,1 /(4 \xi)]$

2./ With $\rho \in R \subseteq\left(0, \rho_{c}:=1\right]$, we had $f(r)=\rho \sin (\pi r) /(\pi r)$, for which $\widehat{f}(\lambda)=$ $\rho \cdot \mathbf{1}(\lambda \in[-\pi,+\pi])$. Thus, $h(r)=-\{\sin (\pi r) /(\pi r)\}^{2}$ and

$$
\begin{aligned}
& \widehat{h}(\lambda)=\frac{-1}{2 \pi}(\mathbf{1}(\lambda \in[-\pi,+\pi]))^{* 2}=\left|\frac{\lambda}{2 \pi}\right|-1 \\
& \widehat{s}(\lambda)=1+\rho\left(\left|\frac{\lambda}{2 \pi}\right|-1\right) .
\end{aligned}
$$

Since $\widehat{s}(\lambda)=1-\rho \geq 0$ for all $\rho \in(0,1]$, we conclude that in this case, $R=$ $\left(0, \rho_{c}:=1\right]$. As announced, we have $\widehat{h}(0)=2 I=\int_{\mathbb{R}} h(r) d r=-1$. When $\rho=\rho_{c}=1$, 
$\widehat{s}(\lambda)=\left|\frac{\lambda}{2 \pi}\right|$ with a linear behavior in a neighborhood of $\lambda=0$. At critical density (corresponding to Dyson gas), as announced, $\sigma_{x}^{2} \sim_{x / \infty} K^{\prime \prime} \log x$.

5./ For $\rho \in R \subseteq\left(0, \rho_{c}:=1\right]$, we had $f(r)=\rho\{\sin (\pi r) /(\pi r)\}^{2}$ with $\widehat{f}(\lambda)=$ $\rho\left(1-\left|\frac{\lambda}{2 \pi}\right|\right)_{+}$. Thus, $h(r)=-\{\sin (\pi r) /(\pi r)\}^{4}$ and, using Theorem 1a of [F], page 28 , it can easily be checked that

$$
\widehat{h}(\lambda)=\frac{-1}{2 \pi}\left(1-\left|\frac{\lambda}{2 \pi}\right|\right)_{+}^{* 2}=\frac{-1}{3 !(2 \pi)^{3}} \sum_{m=0}^{4}(-1)^{m}\left(\begin{array}{c}
4 \\
m
\end{array}\right)(\lambda+(4-2 m) \pi)_{+}^{3} .
$$

We have $\widehat{h}(0)=2 I=\int_{\mathbb{R}} h(r) d r=-2 / 3$.

Although $\widehat{f}(\lambda)$ has compact support, there is no value of the density at which this determinantal point process is super-homogeneous. Indeed, if this were the case, there should be a $\rho_{*}$ at which $1+\rho_{*} \widehat{h}(0)=0$ but since $\widehat{h}(0)=-2 / 3, \rho_{*}=3 / 2$ is outside the range $\rho \in R=\left(0, \rho_{c}:=1\right]$ for which the DPP is well-defined.

6./ With $\rho \in R \subseteq\left(0, \rho_{c}:=1\right]$, we had $f(r)=\frac{2 \rho}{\pi^{2} r^{2}}\left\{\frac{\sin (\pi r)}{\pi r}-\cos (\pi r)\right\}$, for which $f(0)=(2 \rho) / 3$ and circular spectral density: $\widehat{f}(\lambda)=\rho\left(1-\left(\frac{\lambda}{\pi}\right)^{2}\right)_{+}$. Thus, the total correlation function is $h(r)=-\left\{\frac{3}{\pi^{2} r^{2}}\left(\frac{\sin (\pi r)}{\pi r}-\cos (\pi r)\right)\right\}^{2}$ and

$$
\widehat{h}(\lambda)=\frac{-9}{8 \pi}\left(\left(1-\left(\frac{\lambda}{\pi}\right)^{2}\right)_{+}\right)^{* 2} .
$$

After scaling, we get $\left(\left(1-\left(\frac{\lambda}{\pi}\right)^{2}\right)_{+}\right)^{* 2}=\pi k(\lambda / \pi)$ where

$$
k(x)=\int_{\max (-1 ; x-1)}^{\min (1 ; x+1)}\left(1-z^{2}\right)\left(1-(x-z)^{2}\right) d z
$$

which can be integrated to give an exact expression of $\widehat{h}(\lambda)$ and then of the structure factor $\widehat{s}(\lambda)$. From this computation, we easily obtain $\widehat{h}(0)=\frac{-9}{8} k(0)=-6 / 5$. Thus $1+\rho \widehat{h}(0)$ vanishes at density $\rho_{*}=5 / 6$ which is inside the admissible domain for $\rho$, namely inside the interval $\left(0, \rho_{c}:=1\right]$. This shows that, for this DPP, the actual range of $\rho$ for which the PP is well defined is $\left(0, \rho_{*}:=5 / 6\right]$. Further, this DPP is super-homogeneous at $\rho=\rho_{*}$. When $\rho=\rho_{*}$ and when $\lambda$ is close to 0 , we obtain

$$
\widehat{s}(\lambda)=1+\rho_{*} \widehat{h}(\lambda) \sim \frac{3}{2}|\lambda|^{2},
$$

which is locally quadratic. It follows that, in this case, variance saturates with $\sigma_{x}^{2}<K^{\prime}$, as $x \nearrow \infty$. This DPP actually is hyper-uniform at $\rho=\rho_{*}$. When $\rho \in\left(\rho_{*}, \rho_{c}\right]$ this DPP is not defined.

The merits of these examples, if any, are:

Point 1 (from Example 5./). The condition that $\widehat{f}(\lambda)$ is compactly supported is necessary but not sufficient for a translation invariant DPP to be super-homogeneous 
at some admissible value of the density. The DPP of this example is 'essentially Poissonian' in the whole range $\rho \in(0,1]$ for which it is well-defined.

Point 2. (from Example 6./) The condition that $\widehat{f}(\lambda) \in[0,1]$ is necessary but not sufficient for a translation invariant DPP to be well-defined. A translation invariant DPP can start being super-homogeneous at some value of the density which lies inside the admissible domain (and not necessarily at its upper critical bound as in the Dyson gas). This shows that the range $R$ for which the DPP is well-defined can be strictly included in the admissible domain $\left(0, \rho_{c}\right]$.

Point 3. (from Example 6./) There exist translation invariant hyper-uniform DPPs for which the variance saturates. The DPP of Example 6./ at saturation density $\rho=\rho_{*}=5 / 6$ is one of them. We could find no such example in the literature.

\section{Related point processes of interest}

Let $f(r)$ be a continuous real positive-definite function satisfying $f(r)=f(-r)$ with $0<f(0)=\rho<\infty$ and $|f(r) / f(0)| \leq 1$. It follows that $\widehat{f}(\lambda):=\int_{\mathbb{R}} e^{-i \lambda r} f(r) d r$ (which is real) is non-negative for almost all $\lambda$ and that $f(r)=\frac{1}{2 \pi} \int_{\mathbb{R}} e^{i \lambda r} \widehat{f}(\lambda) d \lambda$ is the Fourier transform of the bounded positive spectral density $\hat{f}$. Let $W\left(\mathbf{x}_{n}\right):=$ $\left[f\left(x_{m}-x_{m^{\prime}}\right)\right]_{1}^{n}$ denote the usual $n \times n$ definite-positive weight matrix and let now $\operatorname{Per}\left(W\left(\mathbf{x}_{n}\right)\right)$ stand for its permanent. Then, considering permanents instead of determinants, the point process with correlation functions

$$
\rho_{n}\left(\mathbf{x}_{n}\right)=\operatorname{Per}\left(W\left(\mathbf{x}_{n}\right)\right), n \geq 1
$$

is well-defined and translation invariant, see [HKPV]. It is called a permanental (Boson) point process (PPP).

The generating functional of a permanental point processes reads

$$
\begin{aligned}
\mathbb{E}\left(\prod_{N=1}^{\mathcal{N}(B)}\left(1+z \phi\left(X_{N}\right)\right)\right) & =\sum_{n \geq 0} \frac{z^{n}}{n !} \int_{B^{n}} \prod_{m=1}^{n} \phi\left(x_{m}\right) \cdot \operatorname{Per}\left(W\left(\mathbf{x}_{n}\right)\right) d \mathbf{x}_{n} \\
& =:|I-z W \phi|_{L^{2}(B)}^{-1} .
\end{aligned}
$$

With $W \phi$ is the operator on $L^{2}(B)$ with kernel $W\left(x_{m}, x_{m^{\prime}}\right) \phi\left(x_{m^{\prime}}\right)$, the expansion reduces to the inverse of a Fredholm determinant. PPPs may also be seen as doubly stochastic (Cox) point processes with random intensity given by the squared modulus of a Gaussian process (see [M] and [DVJ], page 172).

\section{Remark:}

Both permanental and determinantal PP are particular cases of $\alpha$-permanental PP where $\alpha \in\{. .,-2,-1\} \cup(0, \infty)$. Indeed, define the $\alpha$-permanent of $W\left(\mathbf{x}_{n}\right)$ by

$$
\operatorname{Per}_{\alpha}\left(W\left(\mathbf{x}_{n}\right)\right):=\sum_{\sigma \in S_{n}} \alpha^{n-c y(\sigma)} \prod_{m=1}^{n} W\left(\mathbf{x}_{n}\right)_{m, \sigma(m)},
$$

where $S_{n}$ stands for the set of permutations of $[n], c y(\sigma)$ the number of cycles of $\sigma \in S_{n}$. The generating functional of a $\alpha$-permanental point processes (for which $\left.\rho_{n}\left(\mathbf{x}_{n}\right)=\operatorname{Per}_{\alpha}\left(W\left(\mathbf{x}_{n}\right)\right), n \geq 1\right)$ reads formally 


$$
\begin{aligned}
\mathbb{E}\left(\prod_{N=1}^{\mathcal{N}(B)}\left(1+z \phi\left(X_{N}\right)\right)\right) & =\sum_{n \geq 0} \frac{z^{n}}{n !} \int_{B^{n}} \prod_{m=1}^{n} \phi\left(x_{m}\right) \operatorname{Per}_{\alpha}\left(W\left(\mathbf{x}_{n}\right)\right) d \mathbf{x}_{n} \\
& =:|I-z \alpha W \phi|_{L^{2}(B)}^{-1 / \alpha},
\end{aligned}
$$

giving respectively permanental and determinantal PP when $\alpha=+1$ and $\alpha=-1$. Conditions under which $\alpha$-permanental PP are well-defined for all $\alpha>0$ seems to be an open problem. $\diamond$

Consider then a PPP with permanental correlation functions $(\alpha=1)$. With $r>0, g(r):=1+\{f(r) / f(0)\}^{2} \in[1,2]$ now is the two-point correlation function of this PPP. The total correlation function is $h(r):=g(r)-1=\{f(r) / f(0)\}^{2}$ which is positive with values in $[0,1]$. In sharp contrast to DPP, translation invariant permanental point processes exhibit clumping as points on the line are now positively correlated. We now give an Example of a 'critical or sub-homogeneous system' for which the structure factor diverges at the origin.

\section{A critical PPP example:}

Let $\alpha \in(0,2]$ and $\zeta>0$. Let $f(r)=\rho\left(1+r^{\alpha}\right)^{-\zeta / 2}$ whose Fourier transform is easily seen to be a positive integrable function. So $f$ is doubly positive. Next, the total correlation function is $h(r)=\left(1+r^{\alpha}\right)^{-\zeta}$, which is also doubly positive. Its Fourier transform $\widehat{h}(\lambda)$ has known closed-form expression depending on $\alpha \zeta<1$, $\alpha \zeta=1$ or $\alpha \zeta>1$. It is known to be a (infinitely divisible) Linnik probability density function (see $[\mathrm{H} 1]$ and $[\mathrm{LL}]$ ), which is positive and integrable. From this, following [LL] for example, with $\gamma$ the Euler constant, we have

$$
\begin{aligned}
& \widehat{s}(\lambda) \sim{ }_{|\lambda| \searrow 0} \frac{\rho}{\cos (\alpha \zeta \pi / 2) \Gamma(\alpha \zeta)}|\lambda|^{-(1-\alpha \zeta)}, \text { if } \alpha \zeta<1 \\
& \widehat{s}(\lambda) \sim{ }_{|\lambda| \searrow 0}-\frac{\rho}{\pi} \log |\lambda|+1-\frac{\gamma \rho}{\pi}, \text { if } \alpha \zeta=1 \\
& \widehat{s}(\lambda) \sim_{|\lambda| \searrow 0} 1+\frac{2 \pi \rho}{\sin ((\zeta-1 / \alpha) \pi)} \frac{\Gamma(1+1 / \alpha)}{\Gamma(\zeta) \Gamma(1-\zeta+1 / \alpha)}, \text { if } \alpha \zeta>1 .
\end{aligned}
$$

When $\delta:=1-\alpha \zeta \in(0,1)$, the structure factor diverges algebraically in a neighborhood of wave-length $\lambda=0$. Consequently, as $x \nearrow \infty$

$$
\sigma_{x}^{2}=\frac{2 \rho x}{\pi} \int_{0}^{\infty}\left\{\frac{\sin (u)}{u}\right\}^{2} \widehat{s}\left(\frac{2 u}{x}\right) d u \sim \kappa x^{2-\alpha \zeta} .
$$

Thus, the variance grows algebraically and faster than volume $x$ (but always slower than $x^{2}$ ). This PPP is a 'critical' or sub-homogeneous system for which the total 2-point correlation function $h$ is long-ranged with $h(r) \sim_{|r| / \infty}|r|^{-\alpha \zeta}$ with exponent smaller than 1 .

When $\alpha \zeta>1$, this PPP is 'essentially (sub-)Poissonian'. When $\alpha \zeta=1, \sigma_{x}^{2} \sim$ $\kappa x \log x$. Variance grows faster than volume with logarithmic corrections to the usual Poisson behavior. 
Tuning down continuously parameter $\zeta$, the status of this PPP shifts from essentially Poissonian to sub-homogeneous with very large window fluctuations, when it crosses the critical value $\zeta_{c}:=1 / \alpha$. We may legitimately ask for the exact role played by parameter $\zeta$. It is related to the infinite divisibility character of the Linnik pdf. In this respect, the following remark may prove helpful.

Remark: Consider a $n \times n$ weight matrix $W\left(\mathbf{x}_{n}\right)$ with $m \times m^{\prime}$ entry: $W\left(\mathbf{x}_{n}\right)_{m, m^{\prime}}=$ $f\left(x_{m}-x_{m^{\prime}}\right)$, defined from definite-positive function $f$ as above. Assume $f$ is also non-negative (and so $f$ is doubly positive). With $\zeta>0$, let $W\left(\mathbf{x}_{n}\right)^{\circ \zeta}$ be the

Hadamard $\zeta$-power of $W\left(\mathbf{x}_{n}\right)$, with entries $\left(W\left(\mathbf{x}_{n}\right)^{\circ \zeta}\right)_{m, m^{\prime}}:=W\left(\mathbf{x}_{n}\right)_{m, m^{\prime}}^{\zeta}$, raising each entry at the power $\zeta$. This new matrix has non-negative entries and, in any case, when $\zeta \geq \zeta_{c}:=n-2$, it remains definite non-negative (see $[\mathrm{FH}]$ ). However, only under some peculiar circumstances is it still a non-negative definite matrix for all $\zeta>0$ (although by Schur theorem it always is when $\zeta$ is an integer). Doubly non-negative matrices whose Hadamard $\zeta$-powers remain doubly non-negative for all $\zeta>0$ are called infinitely divisible matrices (see $[\mathrm{B}]$ ). In the above language to construct definite-positive weight matrices $W$ from doubly positive $f$, it can be checked directly that $W$ is infinitely divisible if and only if $f_{\zeta}(r):=f(r)^{\zeta}$ remains a positive type function for all $\zeta>0$. This clearly is the case for the Linnik example displayed above for which $f_{\zeta}(r)=\rho\left(1+r^{\alpha}\right)^{-\zeta / 2}$. If $f$ fulfills this property, then, while considering its spectral density $\widehat{f}(\lambda)$, for each integer $k$, there is a spectral measure $\widehat{f}_{k}(\lambda)$ such that $\widehat{f}=\widehat{f}_{k}^{* k}$ : In this context, infinite divisibility of $W$ constructed from $f$ as above therefore is the eventual infinite divisibility property of the spectral density $\widehat{f}(\lambda)$ or of $\widehat{h}(\lambda)$ (see $[\mathrm{SVH}]$ ).

We finally observe that super-homogeneous point processes having compactly supported spectral measure, they do not possess this infinite divisibility characteristic. $\diamond$

\section{References}

[1] [B] Bhatia, R. Infinitely divisible matrices. Amer. Math. Monthly, 113, No. 3, 221-235, (2006).

[2] [CL1] Costin, O.; Lebowitz, J.L. Gaussian fluctuation in random matrices. Phys. Rev. Lett., 75, 69 - 72, (1995).

[3] [CL2] Costin, O.; Lebowitz, J. L. On the construction of particle distributions with specified single and pair densities. J. Phys. Chem. B, 108 (51), 19614 -19618, (2004).

[4] [DV] Daley, D. J.; Vesilo, R. Long range dependence of point processes, with queueing examples. Stochastic Process. Appl., 70, no. 2, 265-282, (1997).

[5] [DVJ] Daley, D. J.; Vere-Jones, D. An introduction to the theory of point processes. Springer Series in Statistics. Springer-Verlag, New York, (1988).

[6] [F] Feller, W. An introduction to probability theory and its applications. Vol 2, Wiley, New York, (1971).

[7] [FH] FitzGerald, C. H.; Horn, R. A. On fractional Hadamard powers of positive definite matrices. J. Math. Anal. Appl., 61, No. 3, 633-642, (1977).

[8] [GJL] Gabrielli, A.; Joyce, M.; Labini, F.S. Glass-like universe: Real-space correlation properties of standard cosmological models. Phys. Rev. D, 65, 083523-, (2002).

[9] [GLS] Goldstein, S.; Lebowitz, J. L.; Speer, E. R. Large deviations for a point 
process of bounded variability. Markov Processes and Related Fields, 12, No. 2, 235-256, (2006).

[10] [GO] Godrèche, C.; Oguey, C. Construction of average lattices for quasiperiodic structures by the section method. J. Phys. France, 51, 21-27, (1990).

[11] [GT] Gabrielli, A.; Torquato, S. Voronoi and void statistics for superhomogeneous point processes. Phys. Rev. E, 70, 041105-, (2004).

[12] [H1] Huillet, T. On Linnik's continuous-time random walks. J. Phys. A, 33, No. 14, 2631-2652, (2000).

[13] [H2] Huillet, T. Renewal processes and the Hurst effect. J. Phys. A 35, No. 20, 4395-4412, (2002).

[14] [HKPV] Hough, J. B.; Krishnapur, M.; Peres, Y.; Virág, B. Determinantal processes and independence. Probab. Surv., 3, 206-229, (2006).

[15] [J] Johansson, K. Random matrices and determinantal processes. Les Houches series, Session LXXXIII, Mathematical Statistical Physics, Elsevier, (2006).

[16] [KLS] Kuna, T.; Lebowitz, J.L.; Speer, E.R. Realizability of point processes. Mathematical Physics Preprint Archive, No 06-369, (2006).

[17] [LL] Lim, S.C.; Li, M. A generalized Cauchy process and its application to relaxation phenomena. J. Phys. A, 39, 2935-2951, (2006).

[18] [M] Macchi, O.The coincidence approach to stochastic point processes. Advances in Appl. Probability, 7, 83-122, (1975).

[19] [MS] Meeron, E.; Siegert, A.J.F. Statistical mechanics of hard particle systems. J. Chem. Phys., 48, 3139-3155, (1968).

[20] [S] Soshnikov, A. Determinantal random point fields. (Russian) Uspekhi Mat. Nauk 55 (2000), No. 5(335), 107-160; translation in Russian Math. Surveys, 55, No. 5, 923-975, (2000).

[21] [SVH] Steutel, F. W.; van Harn, K. Infinite divisibility of probability distributions on the real line. Monographs and Textbooks in Pure and Applied Mathematics, 259. Marcel Dekker, Inc., New York, (2004).

[22] [TS] Torquato, S.; Stillinger, F.H. Local density fluctuations, hyperuniformity and order metrics. Phys. Rev. E, 68, 041113-, (2003).

[23] [TW] Tracy, C.A.; Widom, H. Level-spacing distributions and the Airy kernel. Phys. Lett. B, 305, No. 1-2, 115-118, (1993). 